[Vicino Oriente XVI (2012), pp. 245-274]

\title{
TEMPLES ET SANCTUAIRES URBAINS DU LITTORAL SYRIEN \\ A L'AGE DU FER: \\ CONTINUITE ET TRANSFORMATION CULTURELLES
}

\author{
Alice Caltabiano - Université Libre de Bruxelles
}

Recent excavations on Coastal Syria offer new evaluations on Iron Age sacred architecture in Coastal Levant, related to Inner Syrian and progressively to Phoenician tradition, reflecting the regional cultural changes strictly connected to the IA II-III political and economic developments.

Keywords: Syrie côtière; âge du Fer; architecture sacrée; Phéniciens; transformation culturelle

La Syrie côtière, en interface entre le Levant et la Méditerranée, témoigne pendant le I Millénaire av. J.-C. d'un réseau des relations intenses avec la Syrie intérieure, la Phénicie, Chypre et la Grèce, en jouant un rôle important dans les échanges économiques et culturels de la Méditerranée orientale (fig. 1). Les récentes explorations archéologiques et la mise à jour des plusieurs bâtiments religieux offrent une importante contribution pour la compréhension des processus de développement régional durant l' âge du Fer. Le sanctuaire le plus connu dans cette région, le $M a^{\prime}$ abed d'Amrit de la Période Perse ${ }^{1}$, ne constitue en effet qu'une étape avancée de l'architecture du Levant côtier, plus spécifiquement syro-libanaise, en représentant en outre une réalisation très particulière de type monumental et extra-urbain ${ }^{2}$. Vu la rareté des lieux de culte urbains à l' âge du Fer, qui caractérise les centres côtiers, les récentes découvertes ouvrent une intéressante perspective de recherche pour la compréhension de l'architecture religieuse ainsi que des développements culturels dans la côte septentrionale du Levant.

\section{LES DONNÉES ARCHÉOLOGIQUES \\ 1.1. Le temple du Chantier IV à Tell Kazel}

Pendant le Bronze récent et le premier âge du Fer, le cœur religieux de Tell Kazel, ancienne Sumura ${ }^{3}$, est localisé dans le secteur occidental du tell, le Chantier IV, au sud d'une dépression interprétable comme l'entrée ouest de la ville ${ }^{4}$. Selon la séquence architectonique identifiée par l'équipe de l'AUB, le sanctuaire du Fer I est édifié sur les ruines de deux grands bâtiments datés au Bronze récent $\mathrm{II}^{5}$. Après la destruction finale du Bronze récent II, à partir du XI siècle av. J.-C. l'ancien quartier sacré de Tell Kazel est l'objet d'un modeste réaménagement qui, après une première phase de transition structurale

Dunand - Saliby 1985.

On pense aussi au temple de Bostan ech-Cheik à Sidon (Dunand - Saliby 1973; Stucky 1999).

Badre et al. 1990, 17-22.

4 Les fouilles du sanctuaire ont été menées à partir du 1994 par l'American University of Beirut sous la direction de L. Badre, dans le cadre de l'extension et de la compréhension du secteur précédemment investigué par M. Dunand (Dunand - Saliby 1957; Badre - Gubel 1999-2000, 136).

5 Il s'agit du temple du Niveau 6, XIV siècle av. J.-C. (Badre - Gubel 1999-2000: 136, 143-145, fig. 8; Badre 2009, 258-264, fig. 3) et du temple du Niveau 5, XIII-XII siècle av. J.-C. (Badre - Gubel 1999-2000, 170-185, fig. 30; Badre 2009, 264-269, fig. 4). 
(Niveau 4), prévoit la reconstruction d'un petit bâtiment sacré (Niveau 3) ${ }^{6}$, ensuite abandonné au début du Fer $\mathrm{II}^{7}$. Avec des dimensions et des matériaux assez plus modestes que les précédents, les nouvelles structures architectoniques semblent garder toutefois une forte continuité culturelle mise en évidence par la réutilisation du même lieu et, surtout pour ce qui concerne le Niveau 3, par la forte relation des planimétries. La céramique retrouvée dans les couches de destruction et la stratigraphie permettent de considérer les Niveaux 4 et 3 comme deux activités d'édification très proches dont l'arc chronologique peut être fixé entre le XI et le X siècle av. J.-C. .

Le sanctuaire du Fer I à Tell Kazel est représenté par un bâtiment très simple à plan rectangulaire sur un orientation OSO-ENE (fig. 2:1). L'intérieur est occupé principalement par une cella rectangulaire $(17 \times 9 \mathrm{~m})$ érigée en réutilisant en partie les pauvres structures du Niveau 49, mais surtout les ruines du temple du Niveau 5, dont le nouveau sanctuaire reproduit la même orientation et le même plan longitudinal ${ }^{10}$; la cella est probablement caractérisée par deux files de colonnes en bois de soutien pour le toit, placées sur deux bas murets ${ }^{11}$ qui partagent une nef centrale et deux latérales plus petites. Le matériel, récupéré exclusivement à l'intérieur de la cella, est constitué principalement par du mobilier lié

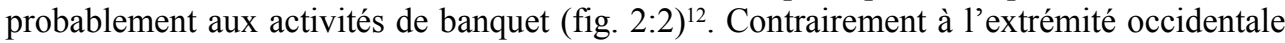
fortement endommagée, l'extrémité orientale du bâtiment est représentée par des murs qui semblent former deux pièces à plan latitudinal reliées au cœur de la fabrique architectonique, même s'ils ne sont pas associés à des sols définis ${ }^{13}$. L'absence de l'entrée complique sans doute la lecture d'ensemble du sanctuaire, mais sur la base de la forte continuité architectonique avec le Niveau 5 on propose de placer l'entrée sur le coté occidental $^{14}$. Sur cette supposition on peut avancer deux reconstructions: un bâtiment

6 Badre - Gubel 1999-2000, 185.

7 La dernière utilisation du sanctuaire (Niveau 3) est scellée par une destruction (Badre - Gubel 1999-2000, 192, 198). Le niveau suivant témoigne d'une occupation sporadique (Niveau 2) représentée seulement par des fosses et siloi (Badre - Gubel 1999-2000, 192, 194, fig. 47) qui précède l'abandon du secteur au IX siècle av. J.-C. et qui a fortement perturbé la conservation des structures.

8 La datation est proposée grâce à la présence de céramique Mycenean IIIC:1b avec des motifs décoratifs en granary style (Badre - Gubel 1999-2000, 189-194, figs. 44, 46), fréquent dans la céramique égéenne seulement à partir du XI siècle av. J.-C. (Killebrew 1998, 163; Lehmann 2007, 490).

9 Les structures du Niveau 4, fortement endommagées, ne permettent pas la reconstruction planimétrique du bâtiment (Badre - Gubel 1999-2000, 185-192, figs. 42-44). Cependant les matériaux associés (fragment de figurine en terre cuite, céramique locale peinte, un gobelet en miniature et une tête de lance en bronze: Badre Gubel 1999-2000, 185, 192, figs. 43-44) ainsi que la continuité avec le Niveau 3, laissent penser à une phase très modeste du lieu de culte.

10 Badre - Gubel 1999-2000, 192-194, figs. 45-46.

11 L'alignement des murs est construit en réutilisant les structures du Niveau 4. La proposition des colonnes est avancée grâce à la découverte de partie de colonnes en bois carbonisées dans la couche de destruction (Badre Gubel 1999-2000, 192).

12 Plusieurs coupes carénées, une cruche trilobée bichrome ainsi qu'un support, une lampe et une coupe tripode en basalte (Badre - Gubel 1999-2000, 192-194, fig. 46:a-h).

13 Badre - Gubel 1999-2000, 192. Cette hypothèse reconstructive donnerait au bâtiment une longueur total de 29 $\mathrm{m}$.

14 Badre - Gubel 1999-2000, 192. Le temple du Niveau 5 est un bâtiment du type Langbau avec entrée in antis sur le coté occidental (Badre - Gubel 1999-2000, 170, 174, fig. 30). 
Langbau à accès direct, comme le temple précédent, ou un édifice à entrée décentrée, cella à trois nefs et niche transversale sur la paroi du fond, qui pourrait évoquer le Temple $2 \mathrm{du}$ sanctuaire pré-phénicien de Kition Kathari, Area II - Floor IV (XIII siècle av. J.-C.) ${ }^{15}$.

Même en l'absence des installations de culte évidentes, l'identification du bâtiment comme un sanctuaire est assurée par le lien fort avec la tradition précédente, comme le témoignent la continuité du lieu de culte, la disposition planimétrique et la superposition de la cella avec celle du complexe du Bronze récent II.

\subsection{Le complexe du Chantier A à Tell Tweini}

Le site de Tell Tweini se dresse dans la Plaine de Jeblè, au milieu de la côte syrienne. Le complexe architectonique du Chantier A est érigé au sommet central du site qui subit une transformation substantielle au début du IX siècle av. J.-C., témoin d'un forte développement urbain ${ }^{16}$ : un quartier d'habitations domestiques du Fer I est partiellement replacé par des bâtiments de nature officielle, destinés à la vie publique de la ville. L'aménagement du quartier central prévoit aussi la construction d'un système routier qui borde le complexe, alors que le quartier d'habitations continue sans interruption à se développer au nord-ouest ${ }^{17}$.

La période relative à l'édification et à la vie du complexe est représentée par la phase Tweini VI de la périodisation de Tell Tweini, qui correspond au Fer II, partagée en deux activités architectoniques ${ }^{18}$. La plus ancienne, représentée par le Niveau $6 \mathrm{CD}$ et datée entre le 900 et le 850 av. J.-C., est indiquée par l'édification de trois bâtiments en proximité19: le Bâtiment A, qui représente l'édifice sacré actuellement le plus connu du complexe, un bâtiment parallèle (Bâtiment B), fouillé partiellement, ainsi qu'un troisième bâtiment encore mal connu (Bâtiment $\mathrm{C}$ ). Une phase plus récente, Niveau 6AB, est représentée par des modifications et adaptations aux bâtiments officiels au cours du VIII siècle av. J.-C., probablement convertis en résidences ${ }^{20}$. Après la destruction du 720 av. J.-C. ${ }^{21}$ ce secteur perd sa connotation monumentale et les bâtiments, ainsi que tout le quartier domestique voisin, sont destinés aux activités industrielles (Niveau 5) ${ }^{22}$.

Le complexe public, détaché des quartiers voisins grâce à la rue conservée au nord-est du Bâtiment $\mathrm{C}$ et au sud du Bâtiment A, se distingue aussi par plusieurs techniques architectoniques et la planimétrie: le deux bâtiments parallèles bordés sur un orientation E$\mathrm{O}$ (Bâtiments A et B) sont probablement identifiables en tant que temples jumeaux, alors que la construction de l'édifice orthogonale en face (Bâtiment C) laisse une petite cour

15 Karageorghis - Demas 1985, 24-37, pls. LXXIX-LXXXIV, plan VIII

16 Probablement en relation avec l'urbanisation des centres araméens (Sader 2000, 75-76; Akkermans - Schwartz 2003, 368).

17 Bretschneider - Van Lerberghe 2008, 43-44; 2010, 43-46, ill. 45, 49; Bretschneider - Van Vyve - Jans 2011, 75, 84-85.

18 Bretschneider et al. 2008, 38-39; Bretschneider - Van Lerberghe 2010, 64, tab. 1.

19 Bretschneider - Van Lerberghe 2008, 44, ill. 54; Bretschneider et al. 2008, 38; Bretschneider - Van Vyve Jans 2011, fig. 8; Vansteenhuyse 2010, 96-98, ill. 2:2-3, 5-7.

20 Bretschneider - Van Lerberghe 2008, 44, ill. 58-59.

21 Bretschneider et al. 2008, 39; Vansteenhuyse 2010, 95-96, ill. 1:7-8.

22 Bretschneider - Van Lerberghe 2008, 44-45, ill. 61; 2010, 47, ill. 52. 
entre les trois bâtiments (fig. 3:1 $)^{23}$. Le Bâtiment A est un édifice rectangulaire (14 x 6,5 $\mathrm{m})$, composé d'une grande pièce longue à plan longitudinale $(8,5 \times 6,5 \mathrm{~m} \mathrm{ca}$.), caractérisé par la saillie de murs latéraux, selon la planimétrie canonique du temple in antis avec cella du type Langraum ${ }^{24}$. L'entrée à accès direct, faite d'un grande dalle régulière, est située sur le coté oriental, alors que sur le coté occidental opposé, une ou deux pièces à plan latitudinale sont accessibles dès la cella. Les techniques de construction témoignent d'une large utilisation de la pierre: le sol de la cella est couvert par un dallage partiellement conservé; les murs maintenus à niveau de fondation sont édifiés en pierre selon une façon très régulière (épaisseur $1,00 \mathrm{~m}$ ) et caractérisés par l'insertion à intervalle régulier de grands blocs carrés (de 2,00 m ca.) dans le corps des murs. Pendant les fouilles les plus récentes, un ensemble de quatre pièces irrégulières mises à jour dans le secteur méridional constitue probablement un secteur d'unités accessoires liées au culte ${ }^{25}$. Le Bâtiment B, dont les fouilles sont encore dans un état préliminaire, présente la même orientation et la même technique de construction que l'édifice précédent, et est formé aussi par une salle allongée ayant un seuil monumental. Parmi le mobilier retrouvé, le plus significatif qui pourrait confirmer la nature religieuse du complexe, est une statuette en bronze de divinité féminine du type chypriote (fig. 3:2) qui remonte au premier âge du $\mathrm{Fer}^{26}$.

\subsection{Le temple dans le Chantier I à Tell Kazel}

La topographie de Tell Kazel subit un changement décisif au moment de transition entre le Fer I et II. Entre la fin du X et le début du IX siècle av. J.-C., probablement en relation avec la pénétration phénicienne dans le site, le quartier sacré de la ville, qui jusqu'à la fin du Fer I est situé dans le secteur occidental (Chantier IV), est déplacé dans le sommet du tell (Chantier I) ${ }^{27}$ remplaçant complètement l'ancien cœur religieux.

Trois phases architecturales principales caractérisent la vie du temple du Chantier I du début du Fer II jusqu'au Fer III: la Phase I (925/900-850 av. J.-C. $)^{28}$ représente l'édification et la première utilisation du temple. La Phase II (850-738 av. J.-C. $)^{29}$ est caractérisée par des aménagements qui modifient légèrement la planimétrie intérieure du temple. La Phase

23 Bretschneider - Van Lerberghe 2008, 44; 2010, 47; Bretschneider - Jans - Van Vyve 2010, 136; Bretschneider - Van Vyve - Jans 2011, 78, 84-85, figs. 6-8.

24 Bretschneider - Van Lerberghe 2008, 44, ill. 55; 2010, 43-44, ill. 40-41, 46; Bretschneider - Jans - Van Vyve 2010, 134-136, ill. 11

25 Les pièces accessoires méridionales sont accolées au mur sud-oriental de la cella et flanquées par la rue qui bord au sud le complexe religieux (Bretschneider - Jans - Van Vyve 2010, 132-134, ill. 13).

26 Bretschneider - Van Lerberghe 2008, ill. 65; 2010, 46, ill. 56.

27 Ce secteur a été fouillé dans les années '50-'60 par M. Dunand et N. Saliby, qui avaient enregistré une grande quantité d'objets votifs (Dunand - Saliby 1957; Dunand - Bounni - Saliby 1964, 5-12, pls. I, VI-VII, XVII, $\mathrm{XX)}$.

28 Gubel 2009a, 455-459. La Phase I correspond aux Niveaux 13-11 de la séquence préliminaire (Badre et al. 1994, 283, fig. 21).

29 Gubel 2009a, 459-464. La Phase II correspond aux Niveaux 10-8 de la séquence préliminaire (Badre et al. 1990, 50-53, figs. 28-29; Badre et al. 1994, 272-283, figs. 11-20; Badre - Gubel 1999-2000, 129-133, figs. 4:e-n, 5). 
III (738-612 av. J.-C.) est enfin identifiée avec un changement fonctionnel du bâtiment qui subit la désacralisation à la suite de la conquête assyrienne dans le 738 av. J.-C. ${ }^{30}$.

\subsubsection{Phase I}

Le projet d'édification original du temple est bien reconnu dans la séquence stratigraphique du Chantier I: après le démantèlement volontaire du quartier domestique du Fer $\mathrm{I}^{31}$, différentes couches de préparation sont placées afin d'obtenir une surface de construction d'environ $200 \mathrm{~m}^{2}$. Le temple installé est un complexe architectural $(18 \times 25 \mathrm{~m}$ ca., sur une orientation NO-SE) (fig. 4:1) partagé en deux secteurs principaux, dont le méridional représente le cœur religieux ${ }^{32}$, et caractérisé par la disposition d'une cella interposée entre deux cours. La cour orientale était destinée probablement à l'accueil des fidèles, qui y déposaient leur offrandes votives. La pièce adjacente allongée représente la cella, pourvue d'un sol couvert de dallage et caractérisée, dans le coin septentrional, par une petite dépression circulaire liée aux activité de libation. La cour occidentale, pavée avec dallage en pente vers l'ouest, semble probablement consacrée aux sacrifices animales (sérail), déposés à l'intérieur des jarres de stockage dans la petite pièce adjacente au nord; l'accès à cette deuxième cour, strictement liée aux activités rituelles, était probablement permis exclusivement au clergé, à travers la cella. Le secteur septentrional est constitué vraisemblablement des unités accessoires, dont la pièce de nord-est, caractérisée par des murs particulièrement élevés, a été interprétée comme une tour mise en relation avec la préparation des gâteaux rituels, selon la tradition retrouvée dans les sources sur la sacralité phénicienne et punique ${ }^{33}$. La localisation de l'entrée au temple, encore inconnue, est supposée par les archéologues en correspondance avec la cour orientale, selon le système de circulation intérieure et grâce à l'identification d'une rue qui flanque le mur méridional du temple ${ }^{34}$.

Du point de vue structural, les murs, construits en brique sous fondations en pierre, sont caractérisés par la mise en place d'orthostates en blocs de pierre carrés aux coins du bâtiment et par l'utilisation de blocs de grès (apparemment importés de la côte syrienne ${ }^{35}$ ) au long des murs périphériques. Des grandes dalles sont utilisées aussi partiellement pour la réalisation des pavements et d'autels rudimentaires. Des autres éléments architectoniques provenant de l'ancien temple du Chantier IV, comme une ancre et une pierre à coupole

30 Gubel 2009a, 464. La Phase III correspond aux Niveaux 7-6 de la séquence préliminaire (Badre et al. 1990, 48-50, plan VI, fig. 26). On propose d'interpréter le grand édifice installé sur les ruines du temple comme la résidence d'un gouverneur installé dans la capitale de la nouvelle province assyrienne (Gubel 2009a, 464).

31 Gubel 2009a, 454-455, fig. 2. Cette phase semble coïncider avec les Niveaux 16-14 du rapport préliminaire (Badre - Gubel 1999-2000, 133-134, fig. 6).

32 Badre - Gubel - Thalmann 2008, 58, fig. 1; Gubel 2009a, 455-457, fig. 3:a-b.

33 Gubel 2009a, 455. Pour la production des gâteaux et plus en général le banquet dans la religion levantine, voir Brelich 1966, 45-50.

34 Probablement la rue sépare le temple d'un autre bâtiment officiel pas encore mis à jour dans toute sa superficie (Gubel 2009a, 455).

35 Une cave d'extraction de grès, contemporaine à la fabrication du temple du Chantier I, est localisée à Tabbat el-Hammam (Braidwood 1940, 204-208, figs. 11-12, 15), notamment identifiée avec le quartier portuaire de Tell Kazel pendant l' âge du Fer (Gubel 2009b). 
insérées dans le dallage d'une des cours, indiquent la volonté de consacrer le nouveau lieu de culte, en essayant de garder une certaine continuité dans la tradition religieuse ${ }^{36}$.

Le mobilier associé au temple de la Phase I (fig. 4:2) est représenté par une attestation significative des supports de culte typiques de la tradition du Fer I-II au Levant ${ }^{37}$, ainsi que des nombreuses figurines en terre cuite du type Breast Astarte, et des figurines masculines en trône avec couronne lebbadé ainsi que des figurines féminines avec tête couronnée à polos, représentant les personnages du clergé ${ }^{38}$. Parmi la production céramique, le plus significatif est une coupe en miniature portant une inscription datée au X siècle av. J.-C. dédiée à $\mathrm{Ba}^{\prime} \mathrm{al}^{39}$.

\subsubsection{Phase II}

La deuxième phase du temple est représentée surtout par le mobilier à destination religieuse, mais le mauvais état de conservation des structures ne permet pas d'arriver à des considérations définitives concernant la nouvelle planimétrie du bâtiment. Le changement le plus évident est apparemment la mise en place d'une couche de grès blanc au-dessus des ruines de la destruction, en particulier sur le mur qui partageait la cella et la cour orientale, en donnant au bâtiment une organisation sur deux cellules adjacentes: une grande cour orientale $^{40}$, et une salle occidentale où 28 jarres retrouvées sur place conservaient les restes brûlés et partagés d'animaux sacrifiés, ainsi que un sceau en bois daté au Fer I, interprété comme un dépôt de refondation ${ }^{41}$. Cette salle occidentale, évidemment destinée aux sacrifices rituels, pourrait représenter le cœur symbolique du temple. Enfin, une concentration de noyaux d'argile pure et de fragments de poterie permet de reconnaître un secteur industriel de production céramique fort lié au lieu de culte, selon une tradition connue dans l'horizon levantin et phénicien ${ }^{42}$. Le mobilier de la Phase II (fig. 4:3) est caractérisé par la continuité des supports de culte, par la présence d'un askos et une jarre phéniciens bichromes datés de la moitié du VIII siecle av. J.-C.43, ainsi que des formes originales red slip en miniature: une sonnette, une gourde et un encensoir ${ }^{44}$. La choroplastique est liée à la tradition de la phase précédente, mais elle est caractérisée par une majeure variété de thèmes, comme la déesse en trône et la dame portant des offres votives $^{45}$.

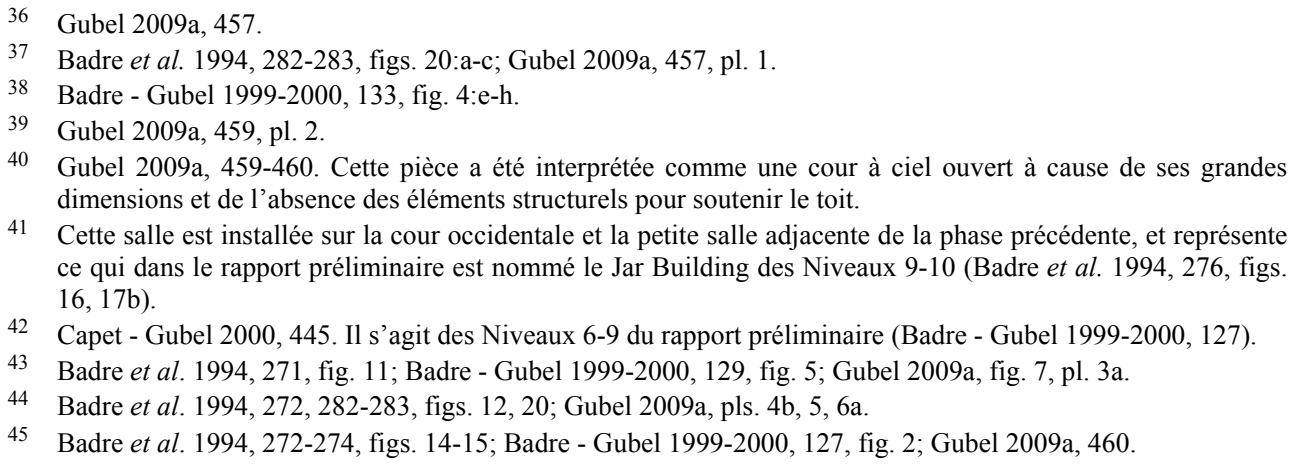

41 Cette salle est installée sur la cour occidentale et la petite salle adjacente de la phase précédente, et représente ce qui dans le rapport préliminaire est nommé le Jar Building des Niveaux 9-10 (Badre et al. 1994, 276, figs. $16,17 \mathrm{~b})$.

42 Capet - Gubel 2000, 445. Il s'agit des Niveaux 6-9 du rapport préliminaire (Badre - Gubel 1999-2000, 127).

43 Badre et al. 1994, 271, fig. 11; Badre - Gubel 1999-2000, 129, fig. 5; Gubel 2009a, fig. 7, pl. 3a.

44 Badre et al. 1994, 272, 282-283, figs. 12, 20; Gubel 2009a, pls. 4b, 5, 6a.

45 Badre et al. 1994, 272-274, figs. 14-15; Badre - Gubel 1999-2000, 127, fig. 2; Gubel 2009a, 460. 
Le mobilier provenant des contextes du temple du Chantier I s'insère à plein titre dans l'horizon cultural phénicien, en faisant du bâtiment de Tell Kazel l'un des temples urbains les mieux investigués de la Phénicie, interprétable comme temple citadin, caractérisé pour toute la période de vie par la distinction des espaces pour l'accueil des fidèles et des espaces destinés aux activités rituelles, dont la cour à ciel ouvert reste un des aspects les plus particuliers.

\subsection{Le sanctuaire occidentale à Tell Arqa}

Le sanctuaire identifié à Tell Arqa, situé à la limite méridionale de la Plain d'Akkar, est constitué d'un bâtiment d'architecture modeste mais articulé. Accolé au rempart qui borde le tell, le petit sanctuaire est érigé à l'extrémité occidentale du tell et bien inséré dans le quartier d'habitations. Même s'il s'agit d'un bâtiment religieux loin des temples monumentaux, il se distingue toutefois des édifices voisins par la planimétrie, ses installations et sa structure architecturale ${ }^{46}$.

L'édification du petit sanctuaire coïncide avec la construction du rempart de la ville au VIII siècle (Niveau 10DC) ${ }^{47}$ et correspond à la période de développement de l'habitat de Tell Arqa pendant le Fer II ${ }^{48}$. L'installation d'un petit groupe de sépultures au dessus du sanctuaire et enfin la couche de destruction attribuée à la conquête assyrienne de la Phénicie septentrionale vers le 740 av. J.-C.49, laissent penser que le lieu de culte a eu une période d'utilisation plutôt limitée à la première moitié du VIII siècle av. J.-C.

La superficie totale du sanctuaire ( $10 \times 25 \mathrm{~m}$ ca., sur une orientation NO-SE) se compose de trois secteurs principaux partagés en unités ouvertes et de salles fermées (fig. $5: 1)^{50}$. Le cœur du bâtiment semble être représenté par une petite salle carrée $(3 \times 4 \mathrm{~m})$ dotée d'un autel en pierre de taille à deux cupules, interprétée comme la cella, et par trois petite pièces connectées qui pourraient représenter des annexes septentrionales pour les activités rituelles. Dans le secteur sud-orientale s'ouvre une cour $(5 \times 7 \mathrm{~m})$, partiellement mise à jour, caractérisée par le sol dallé et la présence de banquettes latérales en brique et pierre, flanquée par deux pièces avec des bassins revêtus d'argile pour les pratiques rituelles. Comme dans le temple du Chantier I à Tell Kazel, la location et les dimensions de la cour méridionale permettent de l'interpréter comme la salle de représentation pour le fidèles qui donnaient leur offrandes, dont une partie a été retrouvée sur l'un des banquettes, et par conséquence comme l'entrée au complexe. Par contre, la destination fonctionnelle de la cour mineure septentrionale reste inconnue, même si par analogie à Tell Kazel elle pourrait être interprété comme une cour privée pour les activités de culte du clergé. Le mobilier du sanctuaire de Tell Arqa consiste principalement en des figurines de terre cuite,

46 Le sanctuaire a été mis à jour dans le Chantier I pendant les fouilles des années '70 par l'équipe francosyrienne menée par J.P. Thalmann (1990; 1998, fig. 1; Badre - Gubel - Thalmann 2008, 58-59, fig. 2).

47 Thalmann 1978, 78, 80-84, 88; 1983, 218; 1990, 50.

48 Thalmann 1983, 217-221.

49 Thalmann 1990, 52-54.

50 Pour la description du sanctuaire voir: Badre - Gubel - Thalmann 2008, 58, fig. 2. 
dont l'iconographie est bien comparable aux exemplaires de Tell Kazel (fig. 5:2) ${ }^{51}$. A la suite de la découverte d'une figurine de déesse assise en trône et parée de symboles astraux ainsi que d'un bloc circulaire réutilisé dans le dallage et gravé d'un signe de Tanit, Thalmann propose d'attribuer le sanctuaire, comme celui de Sarepta ${ }^{52}$, au culte de la déesse Tanit-Astarte ${ }^{53}$.

\subsection{Le Sanctuaire B1 à Tell Tweini}

L'édification du Sanctuaire B1 correspond à un moment très significatif de l'occupation du site, en rapport avec le contexte politique général du Levant et, de l'autre coté, en rapport avec la pénétration de la culture phénicienne. A la fin du VIII siècle av. J.-C. on assiste à un changement important dans l'organisation du site probablement dû à la gestion du territoire lorsque la domination Assyrienne s'établit sur les villes côtières du Levant ${ }^{54}$. A ce moment, on assiste à l'installation des activités artisanales sur le lieu de l'ancien complexe officiel (Chantier A) et en même temps la partie occidentale du tell (Chantier B) héberge un bâtiment religieux qui se distingue des édifices voisins par la planimétrie, la technique et les matériaux de construction. De ce que nous savons actuellement, la phase de construction et d'utilisation du sanctuaire est représentée par le Niveau 6C du Chantier B qui correspond à la phase Tweini VA-B de la périodisation du site et au Fer III de la chronologie syrienne ${ }^{55}$. Le mobilier permet de dater l'utilisation du temple à partir de la fin du VIII siècle av. J.-C. et au VII siècle av. J.-C. ${ }^{56}$.

Le sanctuaire du Chantier B est formé par un complexe architectonique rectangulaire séparé en trois secteurs adjacents qui occupent globalement une superficie de 20 x $10 \mathrm{~m}$ ca. avec orientation nord-sud/est-ouest (fig. 6:1). Le secteur septentrional est occupé par une salle allongée munie d'une structure en calcaire, probablement un autel rudimentaire pour les offres rituelles ${ }^{57}$, communicant avec le noyau du bâtiment: une plateforme avec un sol formé de plaques en pierre $(10.2$ x $4.2 \mathrm{~m})$ flanquée d'une cour orientale à ciel ouvert. Le secteur méridional est caractérisé par des pièces fortement irrégulières pourvues d'installations hydrauliques, et représente probablement des unités accessoires ${ }^{58}$. La disposition générale du bâtiment autour de la grande salle centrale a été classifiée par Bretschneider dans la typologie du broad-room temple ${ }^{59}$.

51 Badre - Gubel - Thalmann 2008, 58. Plusieurs fragments de type de figurines ont été découverts dans les endroits voisins pendant les prospections menées par Chéab dans les années '70 (Chéab 1983, tav. XV:1).

52 Pritchard 1975, 13-40; 1978, 104-108, 132-148.

53 Badre - Gubel - Thalmann 2008, 58.

54 Bretschneider - Van Lerberghe 2010, 47.

55 Il correspond aussi au Niveau 5 du Chantier A (Bretschneider - Van Lerberghe 2010, 64, tab. 1).

56 Bretschneider - Cunningham - Van Lerberghe 1999, 96-98. Les fouilles récentes ont mis à jour une occupation précédente encore inédite (Bretschneider - Van Lerberghe 2010, 64, tab. 1).

57 Bretschneider - Cunningham - Van Lerberghe 1999, 90, figs. 1-2.

58 Bretschneider - Cunningham - Van Lerberghe 1999, 96, figs. 9-10.

59 Bretschneider - Cunningham - Van Lerberghe 1999, 87-104, figs. 1-11, pls. 4-5. 
Plusieurs éléments architecturaux méritent sans doute une mention spécifique. La plateforme centrale $(\mathrm{L} .7500)^{60}(2,90 \times 3,26 \mathrm{~m})$, délimitée par des murs de pierres irrégulières, est réalisée par des grandes dalles en grès importées d'ailleurs et recouvertes par un mince revêtement d'enduit. Un fragment de base de colonne ainsi que le revêtement du sol laissent penser qu'il s'agit d'une salle couverte. A l'intérieur elle est pourvue des deux installations aménagées dans le dallage: une dépression à l'est, identifiable comme un puits sacré, et à l'extrémité septentrionale une installation munie d'un trou circulaire $(18 \mathrm{~cm}$ $\varnothing \times 15 \mathrm{~cm}$ de profondeur), interprété comme la base d'un symbole de culte. La cour orientale (L.7503) est conservée seulement dans sa couche de préparation, pavée de petites pierres $^{61}$, selon une manière identique dans la cour du sanctuaire à Tell Sianu (§ 1.6.). Enfin, la présence significative de l'équipement hydraulique du secteur méridional est sans doute liée à la réception des liquides pour le déroulement des rituels et en même temps pour les autres activités artisanales/industrielles connectées à la vie du sanctuaire. Quelques objets votifs et des restes des offrandes ont été retrouvés dans les environs du bâtiment (fig. 6:2). En particulier dans la salle septentrionale, une offre votive Bucrania a été récupérée à coté de l'autel ainsi qu'un dépôt votif constitué de tessons, os de bovin brulés et un petit ensemble de scories en fer et pointes de flèche ${ }^{62}$, alors que dans la cour orientale, un dépôt de 50 coquillages $^{63}$, peut-être le reste d'un repas rituel. La découverte la plus significative est une figurine en terre-cuite, provenant du secteur méridional, d'un personnage féminin nu avec serpent dans la main, selon un thème connu dans les archives d'Ugarit où la déesse du serpent est dit «fille de la source et de la pierre» ${ }^{64}$. En outre la découverte dans les environs du sanctuaire d'un gourde red slip en miniature ${ }^{65}$, très similaire à l'exemplaire provenant du Temple-Phase II du Chantier I à Tell Kazel, est très remarquable.

Le Sanctuaire B1 de Tell Tweini présente donc une organisation articulée autour de la plateforme destinée aux activités rituelles principales. Même si la planimétrie ne trouve pas de comparaisons exactes, l'éloignement des modèles canoniques à plan axial et l'organisation autour espace ouvert et espaces sacrificiels, permet toutefois de rapprocher ce sanctuaire aux édifices religieux basés sur l'alternance des espaces ouverts et fermés, ainsi que des secteurs pour des activités industrielles.

\subsection{Le Sanctuaire du Chantier D à Tell Sianu}

Etabli dans la partie centrale dominant l'ensemble de la Plaine de Jablé, Tell Sianu représente un site important dans la région en jouant un rôle significatif à l' âge du Fer IIIII ${ }^{66}$. Comme les centres voisins, le site vit un développement urbain décisif au Fer II,

60 Pour la description détaillée de ce secteur voir Bretschneider - Cunningham - Van Lerberghe 1999, 87-90, figs. 4, 6 .

61 La même préparation en pierre est conservée dans d'autres secteurs du sanctuaire, au sud, à l'est et surtout en proximité de la plateforme, peut-être en tant que préparation générale du terrain pour l'édification du bâtiment sacré (Bretschneider - Cunningham - Van Lerberghe 1999, 90, fig. 8)

62 Bretschneider - Cunningham - Van Lerberghe 1999, 90, fig. 7.

63 Bretschneider - Cunningham - Van Lerberghe 1999, 90.

64 Bretschneider - Cunningham - Van Lerberghe 1999, 98, fig. 11:a-c.

65 Al-Maqdissi et al. 2007, fig. 28.

66 Al-Maqdissi 2004; 2008, 62. 
comme le témoigne la construction de la citadelle, partiellement mise à jour dans les secteurs nord-occidental et oriental du tell ${ }^{67}$. A partir du VII siècle av. J.-C., Tell Sianu rentre dans la gestion territoriale de la politique assyrienne, et le site est marqué par un habitat formé des petit habitations ${ }^{68}$. C'est dans ce contexte de type domestique que s'insère le sanctuaire, interprétable comme un sanctuaire de quartier localisé dans le secteur oriental du tell (Chantier D). Le bâtiment est génériquement attribué à la période dite phénicienne tardive, qui correspond à la Phase Sianu IV de la périodisation du site et en générale au Fer III de la chronologie syrienne ${ }^{69}$.

Le sanctuaire est constitué par un bâtiment à plan grossomodo quadrangulaire $(20 \mathrm{x} 22$ m ca.) avec entrée centrale sur le coté méridional (fig. 7). Actuellement le mobilier et sa disposition à l'intérieur de l'édifice ne sont pas encore connus, la destination des espaces peut être reconstruite donc seulement de manière approximative sur la base d'une lecture planimétrique de l'édifice. L'entrée conduit directement dans une cour intérieure qui occupe la majorité de la superficie du sanctuaire, pavée avec des galets de rivière. A l'intérieur de cet espace à ciel ouvert se trouve un autel (en forme de mastaba), et à proximité s'ouvre une petite pièce munie d'une installation centrale, probablement un autel similaire à celui dans la cour, et un bassin sacré ${ }^{70}$. La salle est interprétée par Al-Maqdissi comme une ante-cella ${ }^{71}$, alors que les autres pièces en enfilade du coté occidental pourraient former des annexes pour la conservation des biens liés aux activités religieuses. Les structures architecturales conservées au niveau de fondation ne permettent pas d'identifier les passages ni la connexion entre ces pièces. En particulier, il reste à clarifier s'ils sont tous connectés à la cour sur leur coté oriental ou, selon l'interprétation des unités accessoires, s'ils sont plutôt en communication entre eux et donc accessibles uniquement à travers l'ante-cella grâce à une série d'ouvertures contigues sur un axe nord-sud. Enfin, le coté septentrional du bâtiment est occupé par deux salles respectivement à plan allongé et carré, avec entrées décentrées vers la cour, difficilement interprétables à cause de l'absence, probablement, de quelques installations.

En général, les caractéristiques architecturales du bâtiment portent principalement sur l'organisation autour de la cour intérieure, pavée selon une façon comparable à celle du Sanctuaire B1 à Tell Tweini. D'un point de vue planimétrique, la disposition des petits espaces autour d'une cour centrale majeure semble garder encore une fois la tradition régionale.

\subsection{Le Sanctuaire du secteur nord-oriental à Tell Sukas}

A partir du Bronze récent jusqu'à la période hellénistique le secteur nord-oriental de Tell Sukas est occupé par un espace religieux, dont la continuité permet de l'identifier comme le principal lieu de culte du site. Bien que son organisation ait subi une nette

67 Bounni - Al-Maqdissi 1998, 257-259; Al-Maqdissi 2004, 110.

68 La connaissance en extension est encore limitée (Al-Maqdissi 2009, 331, 335, figs. 4-5; 2010, 325-326, figs. 6-10).

69 Al-Maqdissi 2008, 62; 2010, 321.

70 Al-Maqdissi 2004, 110; 2008, 63.

71 Al-Maqdissi 2008, 63. 
transformation à la fin de l' âge du Fer en correspondance avec l'implantation de la ville gréco-phénicienne, le lien avec la tradition précédente est évident grâce au maintien des installations préexistantes caractérisées par une valeur symbolique et rituelle majeure: un puits circulaire, intégré dans une plateforme plus grande, et l'édification du temple en correspondance avec une cheminée sacrée rudimentaire qui constituait le noyau de l'ancien lieu de culte ${ }^{72}$.

Le sanctuaire gréco-phénicien est un complexe religieux monumental érigé à l'intérieur d'un temenos qui le distingue des quartiers limitrophes et qui englobe plusieurs installations liées à une ou à différentes divinités. Trois phases architectoniques, représentées par la Période G, sont datées entre le début du VII et le début du V siècle av. J.-C. ${ }^{73}$. La Période G3 représente l'édification et la première utilisation (675-588 av. J.-C.); la Période G2 constitue la monumentalisation du complexe à la première moitié du VI siècle av. J.-C. (588-552 av. J.-C.), et la phase finale est représentée par un redimensionnement de la Période G1 Perse (552-498 av. J.-C.). Comme le sanctuaire est largement connu dans la littérature ${ }^{74}$, on se limitera à une brève description du complexe.

\subsubsection{Période G3 (fig. 8:1) (5 $^{75}$}

A l'intérieur du temenos, accessible par une porte principale occidentale, le grand espace à ciel ouvert héberge une terrasse surélevée sur laquelle s'érige le temple, un bâtiment isolé rectangulaire monocellulaire avec orientation ESE-ONO $(7,20 / 35 \mathrm{~m}$ de longueur pour une largeur de 4,32 m à l'est et 3,80 m à l'ouest ${ }^{76}$. L'entrée du temple est située sur le coté oriental, alors que l'extrémité occidentale était occupée par une installation en pierre carrée pourvue de deux trous artificiels, identifiée comme la base d'une statue ou d'un simulacre ${ }^{77}$ (comme dans le Sanctuaire B1 de Tell Tweini). A l'est du temple, le grand autel rectangulaire $(7,35 \times 4,40 \mathrm{~m})$ était édifié en position perpendiculaire au temple, alors qu'au sud une plateforme rectangulaire $(3,7 \times 7,0 \mathrm{~m})$ est construite en englobant et en réaménageant le puits des niveaux précédents ${ }^{78}$. Leurs caractéristiques architectoniques ainsi que les nombreuses traces de cendres, de charbon et les restes d'animaux retrouvés à proximité et sur les deux constructions ${ }^{79}$, donnent la certitude qu'il s'agit respectivement d'installation pour la célébration de sacrifices rituels et pour les activités de libation. A coté des éléments de tradition grecque, comme la localisation

72 Riis 1970, 41, 45. Une probable destination religieuse a été attribuée à ces structures du Complex II-IV dans les Périodes K-J-H (Bronze récent - première âge du Fer) (Riis 1970, 27, 36-40, figs. 7, 11, pl. I).

73 Riis 1970, 126-127. Après un hiatus d'un siècle le sanctuaire est nouvellement reconstruit à la Période Hellénistique (Period F-E) avec un organisation complètement différente (Riis 1970, 92-196, fig. 44).

74 Riis 1970, 40-126; Bonatz 1993, 132-134, fig. 6:4; Mazzoni 2001, 97.

75 Riis 1970, 40-59, fig. 19, pl. III.

76 Riis $1970,42-48$, figs. $12,18$.

77 L'installation mesure $1,20 \times 0,95 \mathrm{~m}$ et des restes of cendres et bois carbonisé on été retrouvés au dessus (Riis 1970, 45, fig. 13)

78 Riis 1970, 41-42, 48-50, fig. 14.

79 Riis 1970, 41. 
perpendiculaire du grand autel et plusieurs éléments de la culture matérielle ${ }^{80}$, la présence de la plateforme à ciel ouvert en relation aux high-places cananéens ${ }^{81}$, ainsi que la continuité d'utilisation du Bronze récent et la localisation dans le cœur de l'habitat indigène $^{82}$, montrent sans doute que le complexe religieux appartient à un horizon culturel mélangé, dans lequel le component levantine joue un rôle assez significatif.

\subsubsection{Période G2}

La planimétrie générale de la deuxième phase architectonique du sanctuaire ne témoigne pas des changements significatifs dans l'organisation des espaces; les modifications substantielles sont représentées par l'agrandissement du temenos et le déplacement de l'entrée principale plus au sud, ainsi que dans la monumentalisation du temple (fig. 8:2) ${ }^{83}$. Le plan du temple est encore de type longitudinale, mais de dimensions plus grandes $(9,90 \times 4,95 \mathrm{~m}$ à l'ouest et 5,30 m à l'est) et à plan tripartite: une entrée in antis avec deux colonnes en pierre ${ }^{84}$ conduisait à une ante-cella pourvue d'une colonne et d'un aménagement circulaire des pierres, pressé dans le sol, qui terminait avec la partie inferieure d'une jarre, constituant probablement une installation pour rituels de libation ${ }^{85}$. La partie occidentale du temple était occupée par la cella, pourvue, comme dans la phase précédente, d'une installation rectangulaire percée $(2,50 \times 1,10 \mathrm{~m})$ au même endroit qu'un puits en entonnoir qui contenait des cendres, probablement un support d'un simulacre divine, mais aussi des os d'animaux et à proximité un couteau de silex utilisé lors des rituels religieux ${ }^{86}$. Le grand autel extérieur est encore en usage sans grand changement, mais son lien avec le temple est souligné par la construction d'une rampe qui connecte désormais les deux structures ${ }^{87}$. Dans le contexte de la monumentalisation du temple, la plateforme ou high-place subit elle aussi une transformation: elle est surélevée et délimitée par une terrasse, mais surtout le puits est fermé et couvert par une couche de pierres qui recouvrent l'entière surface de la plateforme ${ }^{88}$.

Le nouveau complexe présente donc la même organisation générale, mais au niveau symbolique les activités de culte documentent un changement décisif. Si les sacrifices se déroulaient encore dans l'espace à ciel ouvert, soit au-dessus de l'autel soit sur la plateforme, la fermeture du puits extérieur et l'aménagement contextuel des installations dans le temple tripartite, suggèrent au contraire que les rituels de libation se limitaient

80 Riis 1970, 50-57, figs. 15-17. En particulier pour l'organisation des sanctuaires grecs voir: Riis 1970,56 and bibliographie relative au notes 157-163.

81 Riis 1970, 58-59.

82 L'étude de l'habitat démontre que la population d'origine grecque ou chypriote habitait dans les secteurs périphériques du tell, alors que le centre du site, dont l'occupation fixe témoigne d'une continuité depuis l'âge du Bronze, était occupé par la population indigène (Lund 1986, 190).

83 Pour une description détaillée de la Période G2 voir: Riis 1970, 60-87, figs. 20-31, pl. IV.

84 Riis 1970, 62-70, figs. 20, 22-23.

85 Riis 1970,65 , fig. 21.

86 Riis 1970, 64, fig. 30:a

87 Riis 1970, 70-71.

88 Riis 1970, 71-78. 
maintenant à l'espace fermé à l'intérieur du temple et non plus à l'extérieur, probablement en présence exclusive du clergé.

\subsubsection{Période G1}

Après la destruction vers le 553/2 av. J.-C., les restes architectoniques indiquent la reconstruction d'un sanctuaire plus modeste (fig. 8:3). Le temple est replacé par un petit sacellum in antis $(3,30 \times 4,20 \mathrm{~m})$ ouvert sur le coté méridional, de manière similaire à plusieurs petites chapelles édifiées en même temps au Levant côtier, parmi lesquelles la chapelle du bassin sacré d'Amrit ${ }^{89}$ est probablement la plus représentative. Le high-place semble être encore en usage, alors que le grand autel est apparemment abandonné.

\subsection{Le Sanctuaire du port méridional à Tell Sukas}

Bien que le sanctuaire du port méridional de Tell Sukas est un complexe d'Epoque Perse qui sort des limites chronologiques de cet essai, une mention est nécessaire qui complétera la documentation des lieux de culte de la région.

Le sanctuaire se dresse au long de la côte méridionale de la baie sud de Tell Sukas, un secteur qui pendant le Bronze récent et le premier âge du Fer hébergeait un lieu de culte à ciel ouvert ${ }^{90}$, alors que du VII au V siècle av. J.-C. il est occupé par une nécropole à incinération gréco-phénicienne ${ }^{91}$. La reconversion du secteur en sanctuaire est datée de la deuxième moitié du VI siècle av. J.-C., contemporaine aux dernières sépultures sporadiques ${ }^{92}$, et elle est caractérisée par trois phases d'aménagements architectoniques qui rentrent dans les Périodes G1-F-E de la périodisation de Tell Sukas, entre la moitié du VI et le II siècle av. J.-C.93.

Dans la Période G1 le lieu de culte est formé par un petit sacellum in antis dont l'entrée est complètement ouverte dans son coté occidental et probablement surmontée par des merlons, de manière analogue aux petits sacella contemporains du Sanctuaire nord-oriental et à celui dans le bassin d'Amrit (fig. 9:1). Cette structure, nommée Altar Enclosure, contenait deux autels (Altar I, probablement dédié aux offrandes, et Altar II, une sorte d'encensier) ${ }^{94}$ et encerclait le noyau symbolique du lieu de culte: un bétyle déposé dans le terrain, flanqué par une fosse elliptique, probablement un puits rituel pour le déroulement des activités de libation à coté du symbole aniconique de la divinitée ${ }^{95}$. A l'intérieur de l'enclos, plusieurs fragments des figurines constituent la majorité des offrandes ${ }^{96}$.

89 Riis 1970, 88-91, fig. 33, pl. V. Pour Amrit voir: Dunand - Saliby 1985, 31-37, fig. 17.

90 Il s'agit d'un champ des offrandes (Riis et al. 1996, 13-22, pls. II-V). Certaines évidences connexes aux activités de combustion rituelle sont témoignées aussi au Bronze ancien et moyen (Riis et al. 1996, 7, 22-23).

91 Riis 1979, 9-32, pl. II

92 Riis $1979,30$.

93 En général: Riis 1979, 33-68.

94 Riis 1979, 41-43, figs. 127-133.

95 L'interprétation comme puits rituel est soutenue par la présence d'une accumulation de sable sur les parois, preuve d'une activité intense et prolongée du passage de liquides. Le bétyle par contre est constitué d'une pierre naturelle à conformation pyramidale (Riis 1979, 45-46, figs. 142, 143).

96 Il s'agit principalement de statuettes de figures humaines, dont les plus représentatives sont du type d'Héraclès habillé avec peau de lion de la première moitié du V siècle av. J.-C., une figurine en style 
Après une brève période d'abandon, le sanctuaire est agrandi et délimité par un temenos qui englobe l'ancien Altar Enclosure, doté de deux autres autels ${ }^{97}$, et une nouvelle structure édifiée au sud du sacellum, dénommée la Chapel (fig. 9:2). Il s'agit d'un petit bâtiment rectangulaire $(3,50 \times 2,60 \mathrm{~m})$ parallèle au précédent, avec orientation est-ouest, dont l'accès n'est pas conservé ${ }^{8}$. Toutefois on peut observer qu'au même moment de la construction du temenos, les murs nord et sud de l'Altar Enclosure sont prolongés jusqu'au mur du temenos: par conséquence le coté ouest de l'enclos est fermé et, comme le suggère l'organisation générale de l'espace, l'entrée de l'ancienne structure comme celle de la nouvelle Chapel s'ouvrent vers l'est ${ }^{99}$. La construction du mur ouest du temenos provoque la délimitation de l'espace ouvert autour des chapelles religieuses et grâce aux nombreux objets votifs retrouvés dans cet espace ouvert ${ }^{100}$, on peut supposer une entrée principale à l'est directement dans une cour à ciel ouvert pour les fidèles, alors que sur le coté occidental, l'Altar Enclosure et la Chapel se dressait contre le mur du temenos avec accès vers la cour. Les deux chapelles, cœur du sanctuaire, étaient évidemment destinées aux activités rituelles et à la conservation des offrandes, dans lesquels l'accès était permis seulement au clergé. En générale au IV siècle av. J.-C. le sanctuaire subit un aménagement substantiel probablement grâce à la fréquentation intensive d'un lieu de culte érigé en proximité du port. La phase finale du sanctuaire au II siècle av. J.-C. témoigne de plusieurs activités d'aménagement, dont la plus significative est l'agrandissement de l'espace ouvert par le prolongement du mur occidentale du temenos ${ }^{101}$. Dans l'ensemble le champ des offrandes, la présence d'un bétyle et les chapelles renvoient à la série des sanctuaires à ciel ouvert de Byblos, dont le Champ des Offrandes représente le cas le plus explicatif.

\section{CONCLUSION}

Dans l'ensemble de la documentation présentée, un approche diachronique permet d'observer le développement planimétrique des édifices religieux et leur spécificités culturelles. A l'heure actuel, le premier âge du Fer est encore caractérisée par la rareté des témoignages: le seul bâtiment identifié, le temple de Tel Kazel Area IV-Niveau 3, dont le plan n'est pas encore complètement défini, semble faire référence en général à la tradition des temples qui le précèdent au Bronze récent, articulé sur une pièce allongée qui constitue le noyau et presque la totalité du bâtiment. Plus explicitement, les temples successifs à plan longitudinal à Tell Tweini du IX-VIII siècle av J.-C. et à Tell Sukas du VII siècles, témoignent d'une affinité plus constante et durable avec la tradition nord-levantine, évidente par l'adoption du temple in antis et cella de type Langraum $^{102}$, qui restera la

chypriote et un fragment de figure humaine, ainsi que de tessons de céramique grecque et phénicienne (Riis 1979, 40-45, figs. 122-125, 137-141).

97 Riis 1979,44 , figs. 135-136.

98 Riis $1979,33,38$.

99 Riis 1979,51 .

100 Il s'agit encore de figurines en terre cuite de personnage féminin et masculin, dont un temple boy (Riis 1979, 35-36, figs. 99-102, 104-105)

101 Riis 1979, 39, 51-52, fig. 220.

102 Ottosson 1980, 53-60, fig. 9:A-F; Matthiae 1995, 155-157, fig. 35; 2009, 121-125, figs. 7, 11; Wright 1985, 228-237. 
typologie architectonique sacrée la plus répandue et la plus particulière dans la région nordsyrienne pendant toute l' âge du Fer ${ }^{103}$. Avec des minces variations, le plan allongé à accès direct ou tripartite le long de l'axe longitudinale retrouvé dans le temple du Chantier A à Tell Tweini et dans le temple du sanctuaire Nord-oriental à Tell Sukas, est documenté dans les principaux temples monumentaux de la Syrie intérieure contemporaine: le temple de 'Ain Dara ${ }^{104}$, le Building II à Tell Taynat ${ }^{105}$, le Kultraum à Tell Halaf ${ }^{106}$, le Temple A.I à Tell Afis ${ }^{107}$, datés entre le IX et le VII siècle av. J.-C. La relation de Tell Tweini et Tell Sukas, et en général des centres de la plain de Jeblé, avec la Syrie intérieure au IX-VIII siècle av. J.-C. est confirmée aussi par le répertoire céramique qui, contrairement aux sites septentrionaux (comme Al-Mina, Ras el-Bassit, Ras Ibn Hani) et méridionaux (Tel Kazel, Tell Arqa) de la région, est encore fortement lié aux productions nord-syriennes ${ }^{108}$, et spécifiquement de Hama, qui contrôle probablement au Fer II la partie centrale de la Syrie côtière ${ }^{109}$.

Cependant à partir du IX siècle av. J.-C., mais surtout au VIII-VII siècle av. J.-C. la diffusion d'édifices sacrés différents émerge dans la région, à plan apparemment irrégulier ${ }^{110}$ qui ne semble pas répondre à un modèle canonique, mais plutôt partage la même tradition religieuse. Même si certains d'eux nécessitent des recherches ultérieures pour une connaissance plus détaillée, on peut constater qu'ils montrent des caractéristiques communes: tout d'abord l'intégration des espaces à ciel ouvert, notamment des cours qui constituent l'accès principal au sanctuaire et au même temps le lieu de rassemblement des fidèles; ensuite l'existence des installations dédiées aux sacrifices et aux rites de libation ${ }^{111}$, strictement liées aux petites pièces annexes pour la conservation des offrandes ${ }^{112}$, et enfin la présence d'ateliers industriels ${ }^{113}$.

L'usage des cours à l'intérieur des bâtiments religieux est connu au Levant depuis le III Millénaire av. J.-C. ${ }^{114}$, mais dans le cas spécifique, l'organisation des complexes caractérisés par espaces ouverts et par un plan irrégulier est un élément typique de l'architecture sacrée de Byblos. Depuis le Bronze ancien et moyen la présence des cours à ciel ouvert est une particularité des lieux de culte les plus représentatifs et durables de la

103 Perra 1999, 46-50; Mazzoni 2001, 90-93; 2010, 148.

104 Abū 'Assāf 1990; Stone - Zimansky 1999, 4-5.

105 Haines 1971, pl. 103.

106 Von Oppenheim 1950, 357-360, fig. 173.

107 Mazzoni 2010, 145-146, fig. 2.

108 Buhl 1983, 115-117, figs. B-C; Bonatz 1993, 134-144; Vansteenhuyse 2008, 115-118, figs. 10, 12, 15; 2010, 97-99, ill. 3 .

109 Buhl 1983, 117, 124; Bonatz 1993, 155-156; Lund 2004, 77-78; Bretscheider - Van Lerberghe 2008, 43.

110 Cependant ils diffèrent aussi des temples dits à irregular plan identifiés par Mazar dans la Palestine de 1' âge du Fer (Mazar 1980, 62-68, fig. 15)

111 Jidejian 1971, 59-62; Peri 2005; Nigro 2009b, 91-93.

112 Perra 1999, 45-46.

113 Les secteurs industriels liés aux sanctuaires sont connus au Levant, la création et la production étaient considérées comme des activités sacrées (Oggiano 2005, 236). En particulier pour l'horizon phénicien, les ateliers industriels dans les contextes religieux sont bien documentés à Sarepta et à Kition (Floor 2) (Pritchard 1975, 13-14; Wright 1992, 270; Karageorghis [ed.] 2005, 77-79, plan III).

114 Wright 1992, 266; Sala 2008a, 292-299. 
ville, comme le témoignent les complexes du Temple en $\mathrm{L}^{115}$ et le successif Temple des Obélisques ${ }^{116}$ ainsi que les sanctuaires de l'Enceinte Sacrée ${ }^{117}$ et le Champ des Offrandes ${ }^{118}$. $\mathrm{Au}$ Bronze récent une articulation très proche des bâtiments de la Syrie côtière est représentée par le Temple de Kamid el-Lōz, composé de deux cours adjacentes entourées des petites pièces, et caractérisé par la place du sancta sanctorum entre les cours ${ }^{119}$, selon une disposition qui se retrouve de manière plus modeste dans les sanctuaires de Tell Kazel (Chantier I) et Tell Arqa. Dans le I Millénaire les cours à ciel ouvert sont encore un élément important dans l'organisation de l'espace religieux de plusieurs centres phéniciens. Pour citer quelque exemple, à Chypre, à coté de la cour qui précède le Temple monumental d'Astare à Kition-Kathari à partir du IX siècle av. J.-C. ${ }^{120}$, le sanctuaire de KitionBamboula témoigne de la transformation au VI siècle av. J.-C. d'un temple monocellulaire en bâtiment avec des petites pièces et une cour intérieure ${ }^{121}$. Parmi les centre phénicien d'occident, on peut mentionner enfin Motyé, où la phase archaïque du temple dit du Cappiddazzu (VII-VI siècle av. J.-C.) ${ }^{122}$ et le monumental Temple du Kothon daté au VI-V siècle av. J.-C. (Temple $\mathrm{C} 1$ et $\mathrm{C} 2)^{123}$ sont deux bâtiments articulés autour d'un espace central majeur à ciel ouvert. Il est intéressant de noter que dans sa dernière phase de fréquentation le Temple du Kothon est remplacé par un champ des offrandes (Sanctuaire C3) pourvu d'une petite chapelle ${ }^{124}$, comparable au sanctuaire contemporain du Port méridional à Tell Sukas. Lesquels semblent représenter évidemment un héritage plus récent du Champs des Offrandes à Byblos.

Ces témoignages attestent donc l'existence d'une conception commune de l'espace qui, à partir du IX-VIII siècle av. J.-C., trouve sa diffusion et ses propres adaptations dans les régions levantines, cypriote et occidentales de tradition phénicienne. Dans ce contexte, l'architecture religieuse de Byblos, qui connait une forte continuité jusqu'à la Période Perse $^{125}$, se confirme comme le prototype de l'architecture sacrée des centres côtières du Levant et de tradition phénicienne, qui réutilise les espaces ouverts dans l'articulation intérieure des bâtiments religieux urbains, dès ses réalisations monumentales jusqu'aux petits sanctuaires de quartier ${ }^{126}$. Les sanctuaires à cour intérieure érigés dans le milieu urbaine au long de la côte syrienne, semblent donc être fortement influencé par l'architecture religieuse phénicienne, probablement en forte relation avec l'expansion

115 Dunand 1950-1958, 895-898, fig. 1007; Sala 2008b, 69-71.

116 Dunand 1950-1958, 644-552, fig. 767.

117 Dunand 1950-1958, 481, 616-619, 653, 899, pls. XIII:2, XIV, XV; Saghieh 1983, 34-35, 38-39, fig. 11.

118 Dunand 1950-1958, 271-272, 393-399, 899; Saghieh 1983, 30-32, fig. 9, pl. VIII

119 Hachmann 1978a, 10-17, fig. 2; 1978b, 29-34, fig. 1; 1998, 80, fig. 1.

120 Karageorghis (ed.) 2005, 64-89, plan I-IV.

121 Wrigth 1992, 260; Yon 2006, 88-95, figs. 49, 56.

122 Il s'agit du temple archaïque de la phase 2 qui précède la structure monumentale (Nigro 2009a, 244-245, fig. $3)$.

123 Nigro (ed.) 2005, 93-124, pls. IV-VII; Nigro 2009b, 79-85, figs. 5.6, 5.9

124 Nigro (ed.) 2005, 60-92, pls. II-III; Nigro 2009b, 85.

125 Jidejian 1968, 69-70, 96-99; Xella 1994, 206-208.

126 Cette disposition est considérée comme typique dans le monde sémitique, en particulier dans le milieu phénicienne (Wright 1992, 266, 271), et également développée dans le monde punique (Perra 1999, 61). 
commerciale et culturelle des centres phéniciens pendant l' âge du Fer II. En effet ce type de sanctuaire, dont le mobilier est le plus souvent lié à l'horizon phénicien, fait sa première apparition dans le sud de la région analysée, c'est à dire dans les sites de Tell Kazel (Chantier I) et Tell Arqa qui sont plus à coté de la Phénicie propre, et c'est seulement au VII siècle qu'il rejoint la plain septentrionale, notamment Tell Tweini (Sanctuaire B1), autant que Tell Sukas, fortement caractérisé par une réalité culturelle mélangée à la tradition grecque, et Tell Sianu.

Bien que les variations planimétriques montrent des adaptations chaque fois différentes - pour lesquelles il ne faut pas oublier qu'il s'agit en majorité de centres phéniciens mineurs ou qui rentrent dans l'horizon culturel phénicien seulement dans une phase tardive - ces sanctuaires semblent cependant répondre à des nécessités de culte communes. Si d'un coté ils soulignent l'autonomie planimétrique typique de l'architecture phénicienne ${ }^{127}$, libre des canons codifiés de la Syrie contemporaine, en même temps cette indépendance crée un style particulier caractérisé par l'articulation générale des lieux de culte où évidemment les espaces à ciel ouvert et les espaces liés aux sacrifice jouent un rôle de première importance. La diffusion de cette typologie architectonique sur la côte septentrional du Levant, associée au mobilier et aux installations retrouvés dans les sanctuaires, reflète probablement la transformation de la Syrie côtière, dans un premier temps liée à la tradition nord-syrienne, vers un horizon cultural phénicien qui, après la destruction de Hama au 720 av J.-C. ${ }^{128}$, devient particulièrement évident.

\section{BIBLIOGRAPHIE}

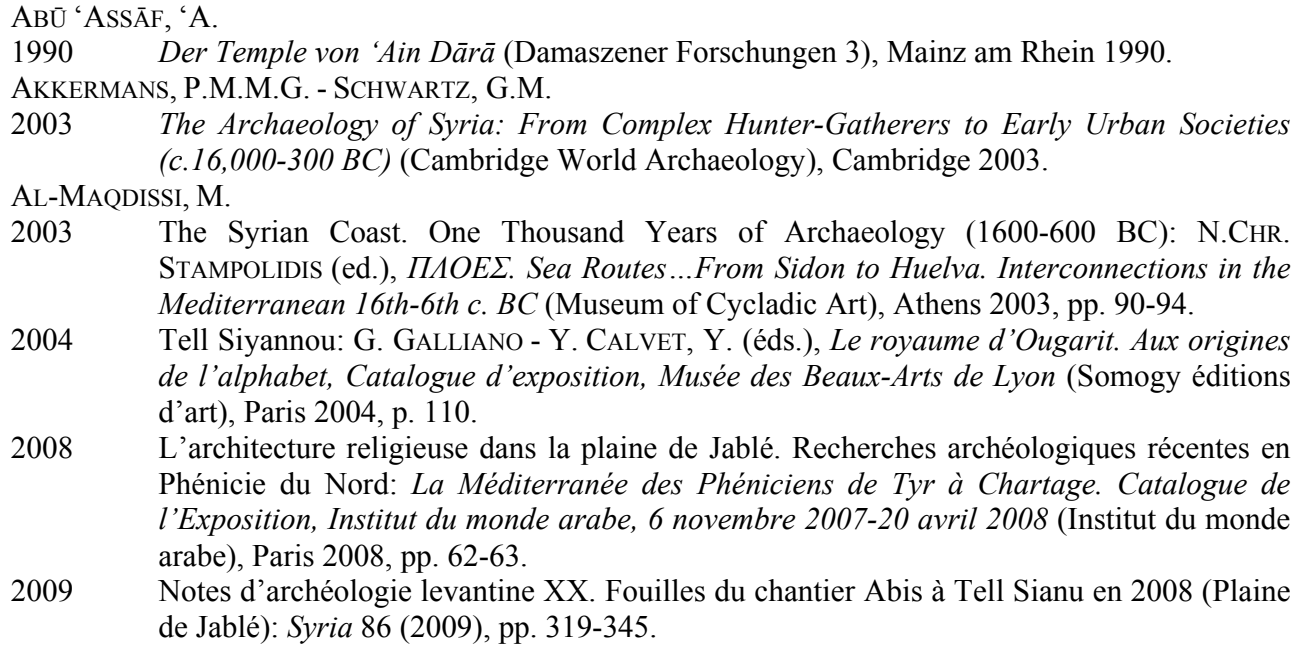

2003 The Syrian Coast. One Thousand Years of Archaeology (1600-600 BC): N.CHR. Stampolidis (ed.), ПИOЕ 2 . Sea Routes...From Sidon to Huelva. Interconnections in the Mediterranean 16th-6th c. BC (Museum of Cycladic Art), Athens 2003, pp. 90-94.

2004 Tell Siyannou: G. Galliano - Y. CALvet, Y. (éds.), Le royaume d'Ougarit. Aux origines de l'alphabet, Catalogue d'exposition, Musée des Beaux-Arts de Lyon (Somogy éditions d'art), Paris 2004, p. 110.

2008 L'architecture religieuse dans la plaine de Jablé. Recherches archéologiques récentes en Phénicie du Nord: La Méditerranée des Phéniciens de Tyr à Chartage. Catalogue de l'Exposition, Institut du monde arabe, 6 novembre 2007-20 avril 2008 (Institut du monde arabe), Paris 2008, pp. 62-63.

2009 Notes d'archéologie levantine XX. Fouilles du chantier Abis à Tell Sianu en 2008 (Plaine de Jablé): Syria 86 (2009), pp. 319-345.

127 Pour une synthèse voir Perra 1999, 44-52, qui soulignait déjà comme les rares sanctuaires phéniciens des contextes non monumentalaux optent pour bâtiments de type «irrégulier».

128 Riis 1948, 194-195. 
2010 Notes d'archéologie levantine XXV. Fouilles du chantier Abis à Tell Sianu en 2009 (Plaine de Jablé): Syria 87 (2010), pp. 319-337.

AL-MAQDISSI, M. ET ALII

2007 Tell Tweini. The Syro-Belgian Excavations (Documents d'Archéologie Syrienne 10), Damas - Leuven 2007.

BADRE, L.

2006 Tell Kazel - Symira: Bulletin of the American Schools of Oriental Research 343 (2006), pp. 65-95.

2009 The Religious Architecture in the Bronze Age: Middle Bronze Beirut and Late Bronze Tell Kazel: Interconnections in the Eastern Mediterranean. Lebanon in the Bronze and Iron Ages. Proceedings of the International Symposium, Beirut 2008 (Bulletin d'Archéologie et d'Architecture Libanaise. Hors-Série VI), Beirut 2009, pp. 253-270.

BADRE, L. ET ALII

1990 Tell Kazel, Syria. Excavations of the Abu Museum, 1985-1987, Preliminary Reports: Berytus 38 (1990), pp. 9-124.

1994 Tell Kazel (Syrie): Rapport Préliminaire sur les $4^{\mathrm{e}}-8^{\mathrm{e}}$ Campagnes des Fouilles (19881992): Syria 71 (1994), pp. 259-346.

BADRE, L. - GUBEL, E.

1999-2000 Tell Kazel, Syria. Excavations of the Abu Museum, 1993-1998. Third Preliminary Report: Berytus 44 (1999-2000), pp. 123-203.

BADRE, L. - GUBEL, E. - THALMANN, J.P.

2008 Trois sanctuaires phéniciens: Sarepta, Tell Arqa, Tell Kazel: La Méditerranée des Phéniciens de Tyr à Chartage. Catalogue de l'Exposition, Institut du monde arabe, 6 novembre 2007-20 avril 2008 (Institut du monde arabe), Paris 2008, pp. 58-59.

BONATZ, D.

1993 Some considerations on the material culture of Coastal Syria in the Iron Age: Egitto e Vicino Oriente 16 (1993), pp. 123-157.

BounNI, A. - AL-MAQDissi, M.

1998 Compte rendu de la cinquième campagne de fouilles à Tell Sianu sur la côte syrienne: Altorientalische Forschungen 25/2 (1998), pp. 257-264.

BRAIDWOOD, R.J.

1940 Report on two sondages on the Coast of Syria, south of Tartous: Syria 21 (1940), pp. 183226.

BRELICH, A.

1966 Introduzione alla storia delle religioni (Edizioni dell'Ateneo), Roma 1966.

BRETSCHNEIDER, J. - CunNingham, T. - VAN LeRBerghe, K.

1999 Gibala: the First two excavations: Ugarit-Forschungen 31 (1999), pp. 75-132.

BRETSCHNEIDER, J. - JANS, G. - VAN VyVE, S.

2010 Les Fouilles du Chantier A en 2009 et 2010. Analyse préliminaire de l'architecture à la transition Bronze Récent - Age du Fer II: M. AL-MAQDisSI - K. VAN LERBERghe - J. BRETSCHNEIDER - M. BADAWY (éds.), Tell Tweini. Onze campagnes de fouilles syro-belges (1999-2010) (Documents d'Archéologie Syrienne), Damas 2010, pp. 131-146.

BRETSChNeIDER, J. - VAN Vyve, S. - Jans, G.

2011 Tell Tweini: A Multi-Period Harbour Town at the Syrian Coast: J. MyNÁRovÁ (ed.), Egypt and the Near East - the Crossroads Proceedings of the International Workshop on the Relations between Egypt and the Near East in the Bronze Age, Prague, September 13, 2010, Prague 2011, pp. 73-87. 
BRETSCHNEIDER, J. - VAN LERBERGHE, K.

2008 Tell Tweini, Ancient Gibala between 2600 B.C.E. and 333 B.C.E: J. BRETSCHNEIDER - K. VAn Lerberghe (eds.), In Search of Gibala, An archaeological and historical study based on eight seasons of excavations at Tell Tweini (Syria) in the A and C fields (19992007) (Aula Orientalis Supplementa 24), Barcelona 2008, pp. 11-68.

2010 Tell Tweini à travers les millenaires: l'histoire et l'archéologie: M. AL-MAQDISSI - K. VAN Lerberghe - J. Bretschneider - M. BADAwy (éds.), Tell Tweini. Onze campagnes de fouilles syro-belges (1999-2010) (Documents d'Archéologie Syrienne), Damas 2010, pp. $15-76$.

BRETSCHNEIDER, J. ET ALII

2008 The Late Bronze and Iron Age in the Jebleh Region: A View from Tell Tweini: H. KÜHNE - R.M. CZICHON - F.J. KREPPNER (eds.), Proceedings of the 4th International Congress of the Archaeology of the Ancient Near East, 29 March - 3 April 2004. Freie Universität Berlin, Wiesbaden 2008, pp. 33-46.

BuHL, M.L.

1983 Sukas VII. The Near Eastern Pottery and Objects of Others Materials from the Upper Strata (Publications of the Carlsberg Expeditions to Phoenicia 9), Copenhagen 1983.

CAPET, E. - GuBEL, E.

2000 Tell Kazel - Six Century of Iron Age Occupation (c. 1200-612 B.C.): G. BunNENS (ed.), Essay on Syria in the Iron Age (Ancient Near Eastern Studies Supplementum 7), Louvain CHÉAB, M. - Paris - Sterling 2000, pp. 425-457.

1983 Découvertes phéniciennes au Liban: P. BARTOLONI - S.F. BONDÌ - G. COACCI POLSELLI M.T. Francisi - F. Mazza - G. Petruccioli - P. Xella (a cura di), Atti del I Congresso Internazionale di Studi Fenici e Punici, Roma, 5-10 Novembre 1979, Roma 1983, pp. $165-172$.

DUNAND, $\mathrm{M}$.

1950-1958 Fouilles de Byblos. Tome II. 1933-1938 (Études et documents d'archéologie III), Paris 1950-1958.

DunAND, M. - BounNi, A - SALiBY, N.

1964 Fouilles de Tell Kazel, rapport préliminaire: Annales Archéologiques Arabes-Syriennes 14 (1964), pp. 1-14.

DUNAND, M. - SALIBY, N.

1957 A la recherche de Simyra: Annales Archéologiques Arabes-Syriennes 7 (1957), pp. 3-16.

1973 Le temple d'Echmoun à Sidon: Bullettin du Musée de Beyrouth 26 (1973), pp. 7-25.

1985 Le temple d'Amrith dans la Pérée d'Aradus (Bibliothèque Archéologique et Historique 121), Paris 1985.

GuBEL, E.

2009a The Phoenician Temple at Tell Kazel (Șumur): Interconnections in the Eastern Mediterranean. Lebanon in the Bronze and Iron Ages. Proceedings of the International Symposium, Beirut 2008 (Bulletin d'Archéologie et d'Architecture Libanaise. Hors-Série VI), Beirut 2009, pp. 453-468.

2009b Phoenician Towns and Harbours in the North. The Case of Iron Age Sumur (Tell Kazel), Syria: S. Helas - D. Marzoli (Hrsg.), Phöinizisches und punisches Städtewesen. Akten der internationalen Tagung in Rom vom 21. bis 23. Februar 2007 (Iberia Archaeologica, Band 13), Mainz am Rhein 2009, pp. 45-54. 
HACHMANN, R.

1978a Rapport préliminaire sur les fouilles au Tell de Kamid el-Loz de 1969 à 1972, avec appendice concernant des découvertes épigraphiques par G. Wilhelm: Bulletin du Musée de Beyrouth 30 (1978), pp. 7-26.

1978b Rapport préliminaire sur les fouilles au Tell de Kamid el-Loz en 1973: Bulletin du Musée de Beyrouth 30 (1978), pp. 27-43.

1998 Kamid el-Loz: métropole de la Beqaa: Liban, l'autre rive. Catalogue de l'Exposition présentée à l'Institut du monde arabe du 27 octobre 1998 au 2 mai 1999 (Institut du HAINES, R.C. monde arabe), Paris 1998, pp. 78-80.

1971 Excavations in the Plain of Antioch, II. The Structural Remains of the Later Phases. Chatal Hüyük, Tell Al-Judaidah and Tell Ta'ynat (Oriental Institute Publications 95), Chicago 1971.

JIDEJIAN, N.

1968 Byblos Through the Ages, Beirut 1968.

$1971 \quad$ Sidon Through the Ages, Beirut 1971.

KARAGEORGHIS, V. (ed.)

2005 Excavations at Kition VI. The Phoenician and Later levels, Part I-II (Department of Antiquities), Nicosia 2005.

Karageorghis, V. - Demas, M. (eds.)

1985 Excavations at Kition V. The Pre-Phoenician Levels, Areas I and II (Department of Antiquities), Nicosia 1985.

KILLEBREW, A.E.

1998 Mycenaean and Aegean-Style Pottery in Canaan during the $14^{\text {th }}-12^{\text {th }}$ centuries B.C.: E.H. Cline - D. HARris-Cline (eds.), The Aegean and the Orient in the Second Millennium. Proceedings of the 50 ${ }^{\text {th }}$ Anniversary Symposium Cincinnati, 18-20 April 1997 (Annales d'Archéologie égéenne de l'Université de Liège 18), Liège 1998, pp. 159-169.

LEHMANN, G.

2007 Decorated Pottery Styles in the Northern Levant during the Early Iron Age and their Relationship with Cyprus and the Aegean: Ugarit Forschungen 39 (2007), pp. 487-529.

LUND, J.

1986 Sūkās VIII. The Habitation Quarters (Publication of the Carlsberg Expedition to Phoenicia 10), Copenhagen 1986.

2004 The Iron Age and the Graeco-Roman Period: J.P. RIIS - I. THUESEN - J. LuND - T. RIIS, Topographical Studies in the Gabla Plain (Publication of the Carlsberg Expedition to Phoenicia 13), Copenhagen 2004, pp. 38-84.

MatThiae, P.

1995 Ebla. Un impero ritrovato, Torino 1995.

2009 Temples and Queens at Ebla. Recent Discoveries in a Syrian Metropolis between Mesopotamia, Egypt and Levant: Interconnections in the Eastern Mediterranean. Lebanon in the Bronze and Iron Ages. Proceedings of the International Symposium, Beirut 2008 (Bulletin d'Archéologie et d'Architecture Libanaise. Hors-Série VI), Beirut 2009, pp. 117-139.

MAZAR, A.

1980 Excavations at Tell Qasile. The Philistine Sanctuary: Architecture and Cult Objects (Qedem Monographs of the Institute of Archaeology of Jerusalem 12), Jerusalem 1980. MAZZONI, S.

2001 Temples in the City and the Countryside: New Trends in Iron Age Syria: Damaszener Mitteilungen 13 (2001), pp. 89-99. 
2010 Santuari a Tell Afis nell'età del Ferro (I mill. a.C.) e l'architettura templare del Levante: G. Bartoloni - P. Matthiae - L. Nigro - L. Romano, Tiro, Cartagine, Lixus: nuove acquisizioni, Atti del Convegno Internazionale in onore di Maria Giulia Amadasi Guzzo, Roma, 24-25 novembre 2008 (Quaderni di Vicino Oriente 4), Roma 2010, pp. 143-161.

NigRo, L.

2009a Il Tempio del Kothon e il ruolo della aree sacre nello sviluppo urbano di Mozia dall'VIII al VI secolo a.C.: S. Helas - D. MARzoli (Hrsg.), Phöinizisches und punisches Städtewesen. Akten der internationalen Tagung in Rom vom 21. bis 23. Februar 2007 (Iberia Archaeologica, Band 13), Mainz am Rhein 2009, pp. 241-270.

2009b Il Tempio del Kothon e le origini fenicie di Mozia: A. MAStino - P.G. Spanu - R. ZuccA (eds.), Naves plenis velis euntes (Tharros Felix 3), Roma 2009, pp. 77-118.

NigRO, L. (ed.)

2005 Mozia - XI. Zona C, il Kothon. Rapporto preliminare delle campagne di scavo XXIII e XXIV (2003-2004), condotte congiuntamente con il Servizio Beni Archeologici della Soprintendenza Regionale per $i$ Beni Culturali e Ambientali di Trapani (Quaderni di Archeologia Fenicio Punica II), Roma 2005.

OGGIANO, I.

2005 Dal terreno al divino. Archeologia del culto nella Palestina del primo millennio, Roma 2005.

OTTOSSON, M.

1980 Temples and Cult Places in Palestine (Uppsala Studies in Ancient Mediterranean and Near Eastern Civilizations 12), Uppsala 1980.

PERI, C.

2005 La roccia e il diluvio: considerazioni sul tempio siro-palestinese: A. SpANò GiAmMELLARO (a cura di), Atti del V Congresso Internazionale di Studi Fenici e Punici, MarsalaPalermo, 2-8 ottobre 2000 (Università degli Studi di Palermo), Palermo 2005, pp. 145148.

PERRA, C.

1999 Sulle origini dell'architettura templare fenicia e punica di Sardegna: Rivista di Studi Fenici 27/1 (1999), pp. 43-77.

PRITCHARD, J.B.

1975 Sarepta. A Preliminary Report on the Iron Age. Excavations of the University Museum of the University of Pennsylvania, 1970-1972 (Museum Monographs), Philadelphia 1975.

1978 Recovering Sarepta, A Phoenician City, Princeton 1978.

RIIS, P.J.

1948 Hama. Fouilles et recherches de la Foundation Carlsberg 1931-1938. Volume II 3: Les cimetières à crémation (Nationalmussets Skrifter, Større Beretninger 1), Copenhagen 1948.

1970 Sükās I. The North-East Sanctuary and the First Settling of Greeks in Syria and Palestine (Publications of Carlsberg Expedition to Phoenicia 1), Copenhagen 1970.

1979 Sükās VI. The Greco-Phoenician Cemetery and Sanctuary at the Southern Harbour (Publication of the Carlsberg Expedition to Phoenicia 7), Copenhagen 1979.

RIIS, P.J. ET ALII

1996 Sükās X. The Bronze and Early Iron Age Remains at the Southern Harbour (Publications SADER, $\mathrm{H}$. of Carlsberg Expedition to Phoenicia 12), Copenhagen 1996.

2000 The Aramean Kingdoms of Syria. Origin and Formation Processes: G. BunNENS (ed.), Essay on Syria in the Iron Age (Ancient Near Eastern Studies Supplementum 7), Louvain - Paris - Sterling 2000, pp. 61-76. 
SAGHIEH, M.

1983 Byblos in the Third Millennium B.C., Warminster 1983.

SALA, M.

2008a L'architettura sacra della Palestina nell'età del Bronzo Antico I-III. Contesto archeologico, analisi architettonica e sviluppo storico (Contributi e Materiali di Archeologia Orientale XIII), Roma 2008.

2008b Il Temple en L a Biblo: Vicino Oriente XIV (2008), pp. 61-87.

STONE, E - ZiMANSKY, P.

1999 The Iron Age Settlement at 'Ain Dara, Syria: Survey and Soundings (British STUCKY, R. Archaeological Reports. International Series 786), Oxford 1999.

1999 Le temple d'Echmoun à Bostan ech-Cheikh: Liban, l'autre rive. Catalogue de l'Exposition présentée à l'Institut du monde arabe du 27 octobre 1998 au 2 mai 1999 THALMANN, J.P. (Institut du monde arabe), Paris 1998, pp. 133-138.

1978 Tell 'Arqa (Liban Nord). Campagnes I-III (1972-1974), Rapport Préliminaire: Syria 55 (1978), pp. 1-151.

1983 Les Niveaux de Age du Bronze et de l'Age du Fer à Tell 'Arqa (Liban): P. BARTOLONI S.F. Bondì - G. CoAcCi Polselli - M.T. Francisi - F. MAZZA - G. Petruccioli - P. Xella (a cura di), Atti del I Congresso Internazionale di Studi Fenici e Punici, Roma, 5-10 Novembre 1979, Roma 1983, pp. 217-222.

1990 Tell 'Arqa, de la conquête assyrienne à l'époque perse: Transeuphratène II (1990), pp. 51-57.

1998 Deux sanctuaires phéniciens: Arqa et Sarepta: Liban, l'autre rive. Catalogue de l'Exposition présentée à l'Institut du monde arabe du 27 octobre 1998 au 2 mai 1999 (Institut du monde arabe), Paris 1998, p. 132.

VANSTEENHUYSE, $\mathrm{K}$.

2008 The ceramic material from Field A: J. BRETSCHNEIDER - K. VAN LERBERGHE (eds.), In Search of Gibala, An archaeological and historical study based on eight seasons of excavations at Tell Tweini (Syria) in the A and C fields (1999-2007) (Aula Orientalis Supplementa 24), Barcelona 2008, pp. 103-140.

2010 La céramique de chantier A: M. AL-MAQDISSI - K. VAN LERBERGHE - J. BRETSCHNEIDER M. BADAWY (éds.), Tell Tweini. Onze campagnes de fouilles syro-belges (1999-2010) (Documents d'Archéologie Syrienne), Damas 2010, pp. 95-114.

VON OPPENHEIM, M.F.

$1950 \quad$ Tell Halaf, Zweiter Band: Die Bauwerke, Berlin 1950.

WRIGHT, G.R.H.

1985 Ancient Building in South Syria and Palestine (Handbuch der Orientalistik 3), Leiden Köln 1985.

1992 Ancient Building in Cyprus (Handbuch der Orientalistik 8), Leiden - New York - Köln 1992.

XELla, P.

1994 Pantheon e culto a Biblo. Aspetti e problemi: E. ACQuARO - F. MAZZA - S. RiBichini - G. SCANDONE - P. Xella (a cura di), Biblo. Una città e la sua cultura (Collezione di Studi Fenici 34), Roma 1994, pp. 195-214.

YON, $\mathrm{M}$.

2006 Kition de Cypre (Recherche sur les civilisations. Guides archéologiques de l'Institut français d'archéologie du Proche-Orient 4), Paris 2006. 


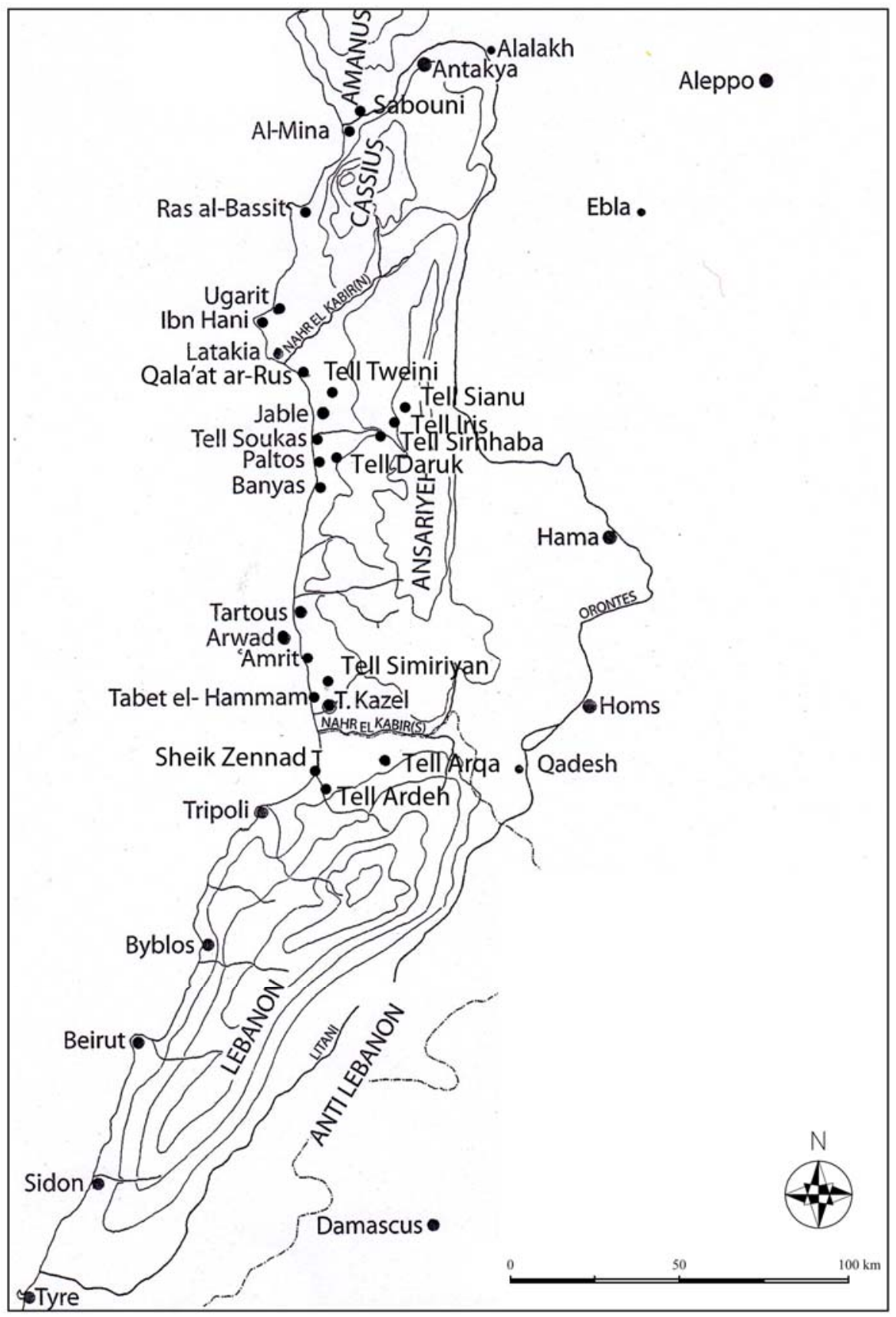

Fig. 1: Carte archéologique de la Syrie côtière (après Badre 2006, fig. 1). 

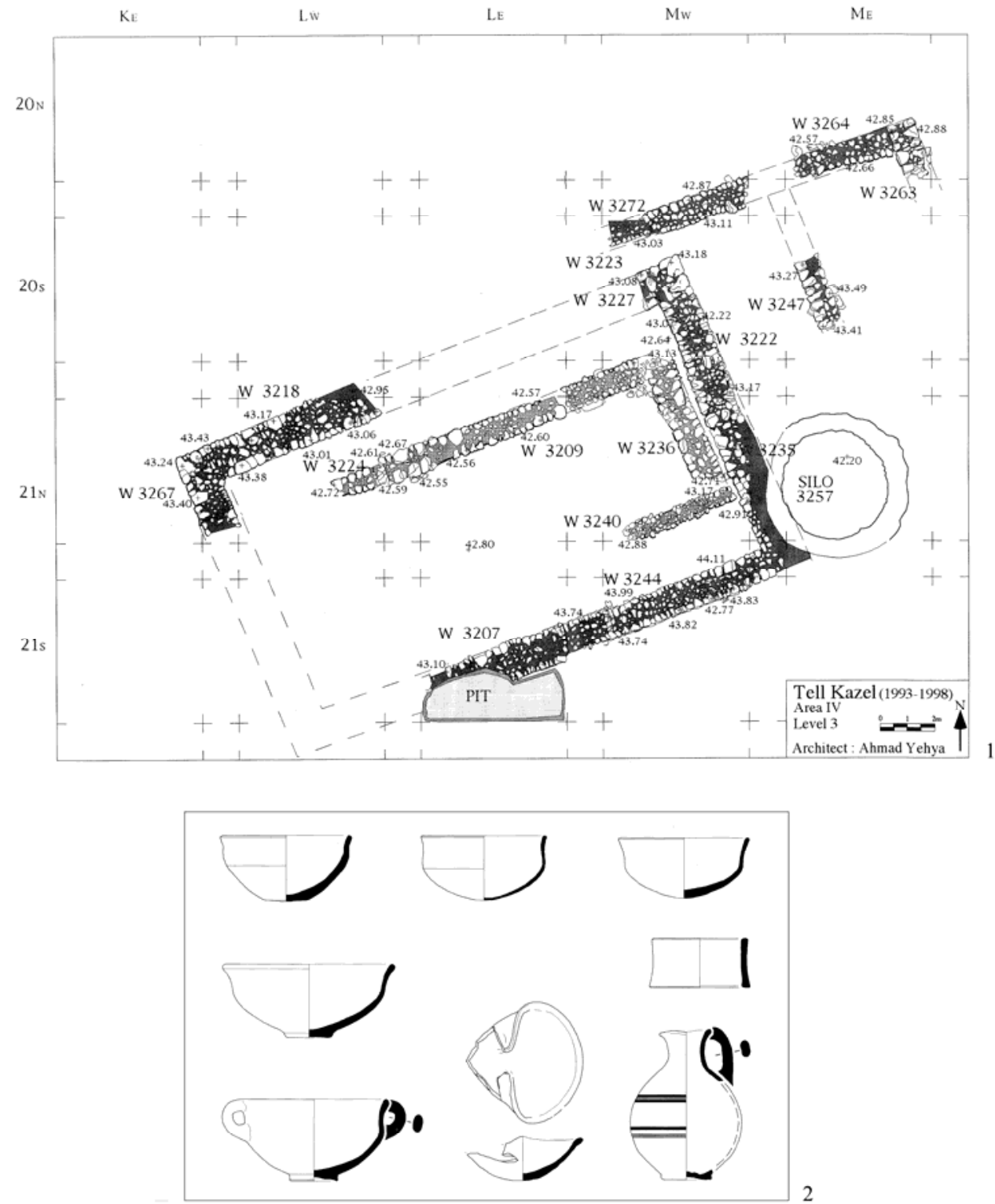

Fig. 2: Tell Kazel, Chantier IV, Niveau 3. 1, plan du temple; 2, mobilier provenant de la cella; XI-X siècle av. J.-C. (après Badre - Gubel 1999-2000, figs. 45-46). 

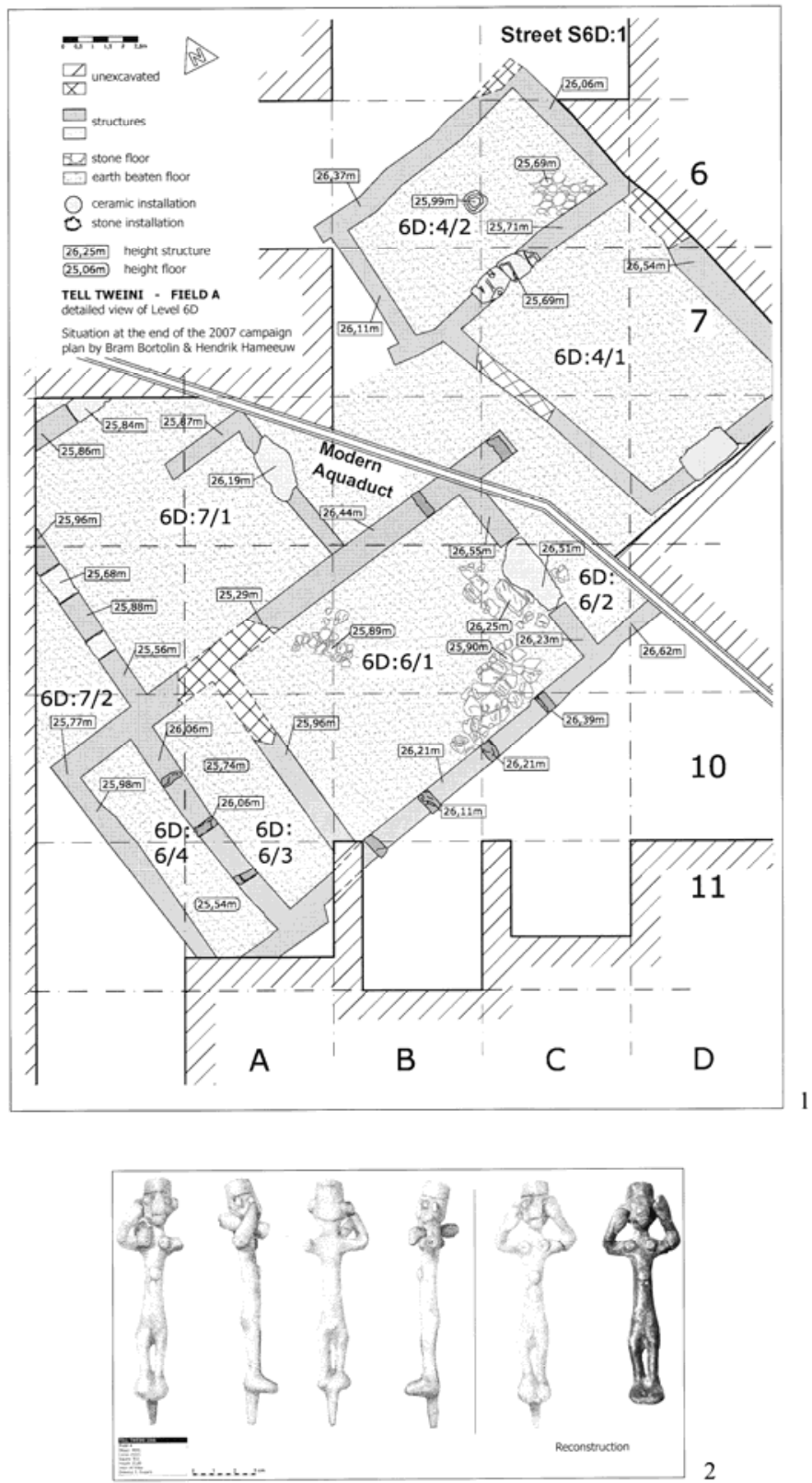

Fig. 3: Tell Tweini, Chantier A, Niveau 6CD. 1, plan des Batiments A, B et C; 2, figurine en bronze provenant du Bâtiment A; 900-850 av. J.-C. (après Bretschneider - Van Lerberghe 2008, ill. 55, 65). 

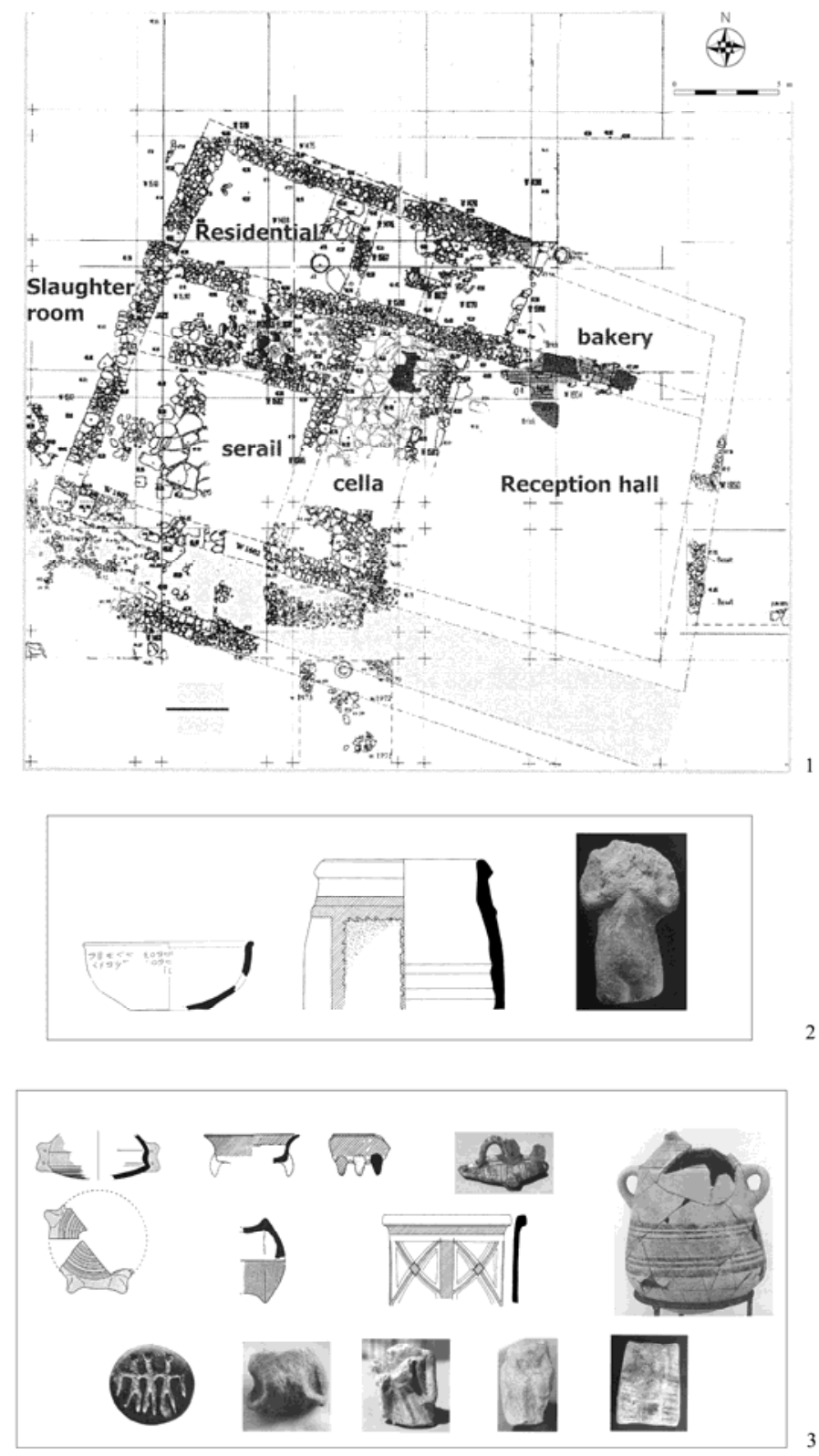

Fig. 4: Tell Kazel, Chantier I; 1-2, plan du temple et mobilier, Phase I; 925/900-850 av. J.C. (après Gubel 2009a, figs. 3b, 4, pls. 1-2); 3, mobilier provenant du temple de la Phase II; 850-738 av. J.-C. (après Capet - Gubel 2000, figs. 20, 23-25; Gubel 2009a, fig. 7, pls. 4a, $5)$. 

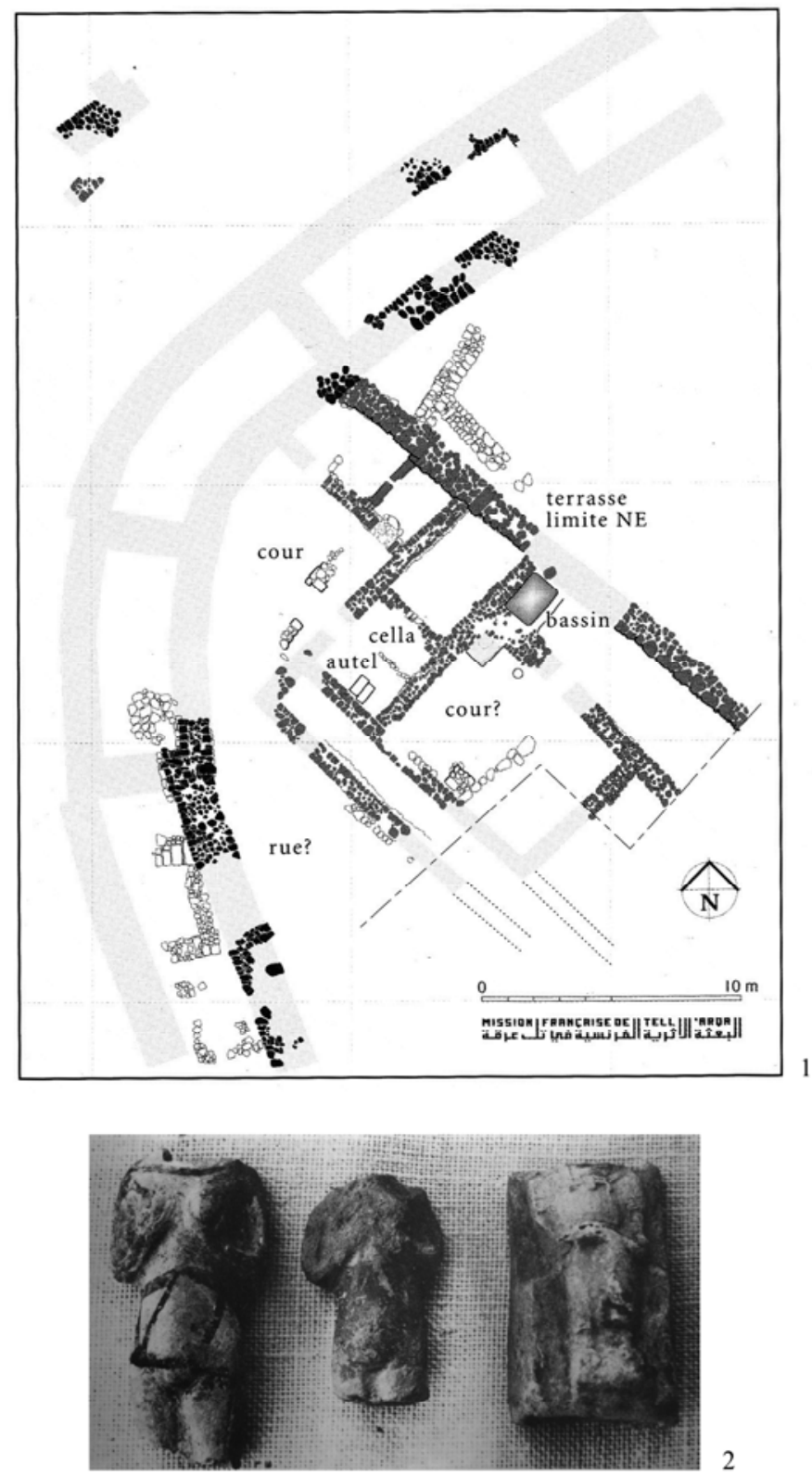

Fig. 5: Tell Arqa, Chantier I, Niveau 10DC; 1, plan du sanctuaire; première moitié VIII siècle av. J.-C. (Thalmann 1998, fig. 1); 2, figurines en terre cuite provenant du secteur occidental du tell (Chéab 1983, pl. XV:1). 

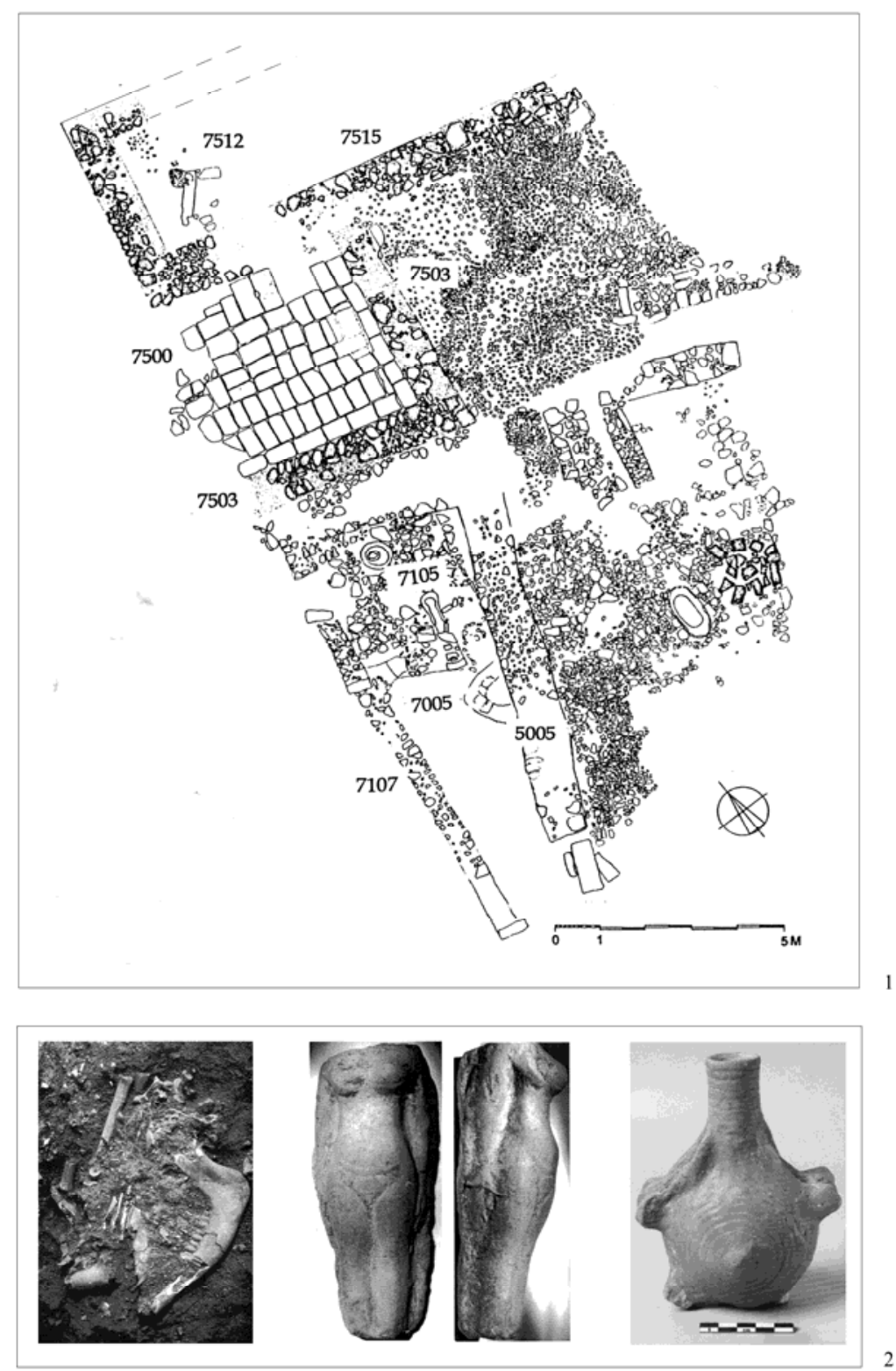

Fig. 6: Tell Tweini, Chantier B, Niveau 6C; 1, plan du Sanctuaire B1 (Bretschneider Cunningham - Van Lerberghe 1999, pl. 4); 2, offrandes et objets provenant du Sanctuaire B1; fin VIII - début du VII siècle av. J.-C. (après Bretschneider - Cunningham - Van Lerberghe 1999, figs. 10-11; Al-Maqdissi et al. 2007, fig. 31). 
Fig. 7: Tell Sianu, Chantier D, Sianu IV. Plan du sanctuaire; VII siècle av. J.-C. (après Al-Maqdissi 2003, fig. 4).
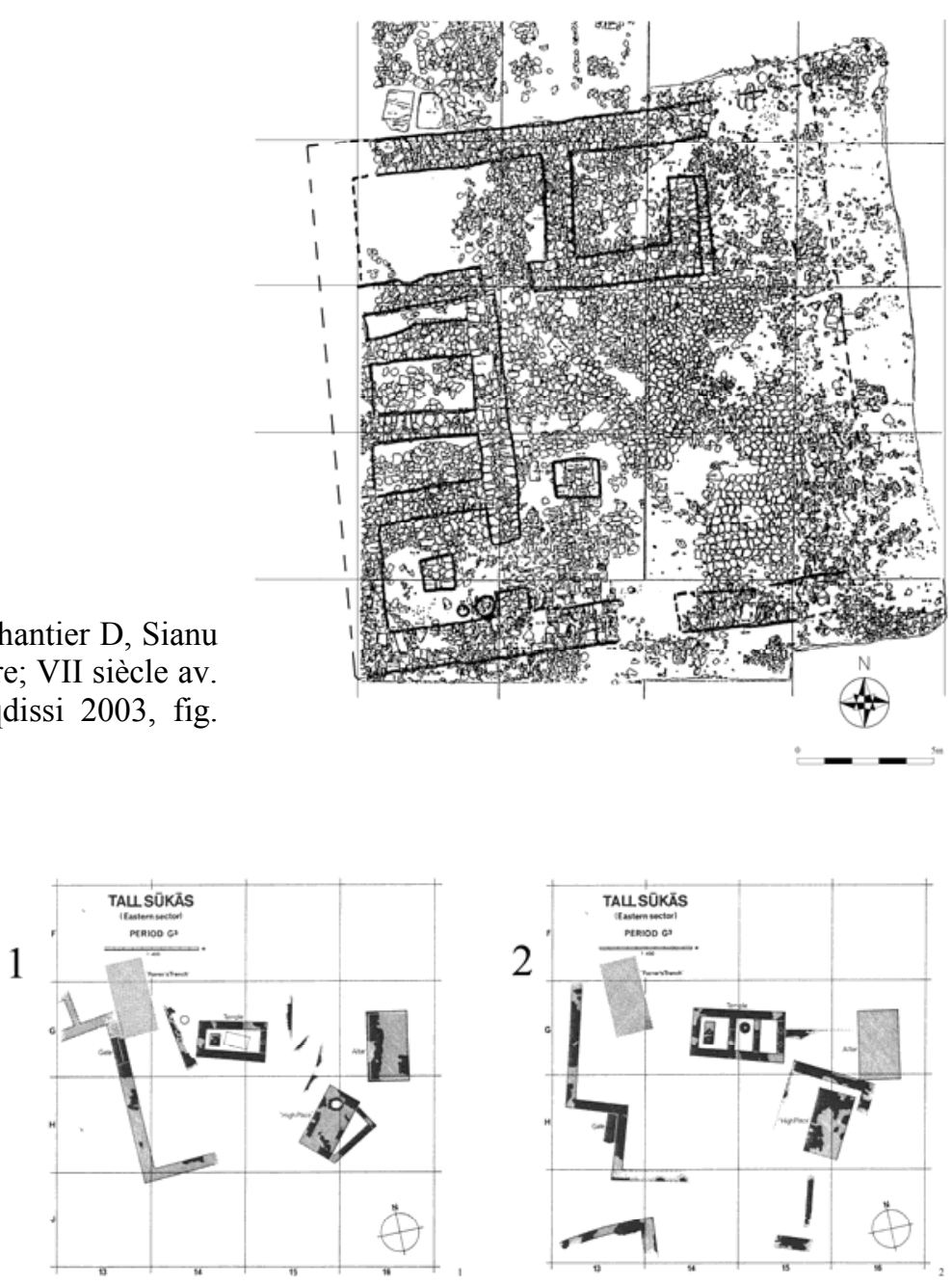

Fig. 8: Tell

Sukas, Secteur nord-oriental; 1-3, séquence architectonique du sanctuaire dans les Periodes G3-G1, VII-VI siècle av. J.-C. (après Riis 1970, figs. 19, 31, 33).

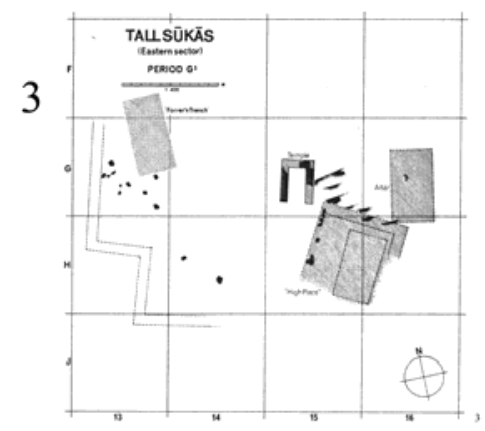



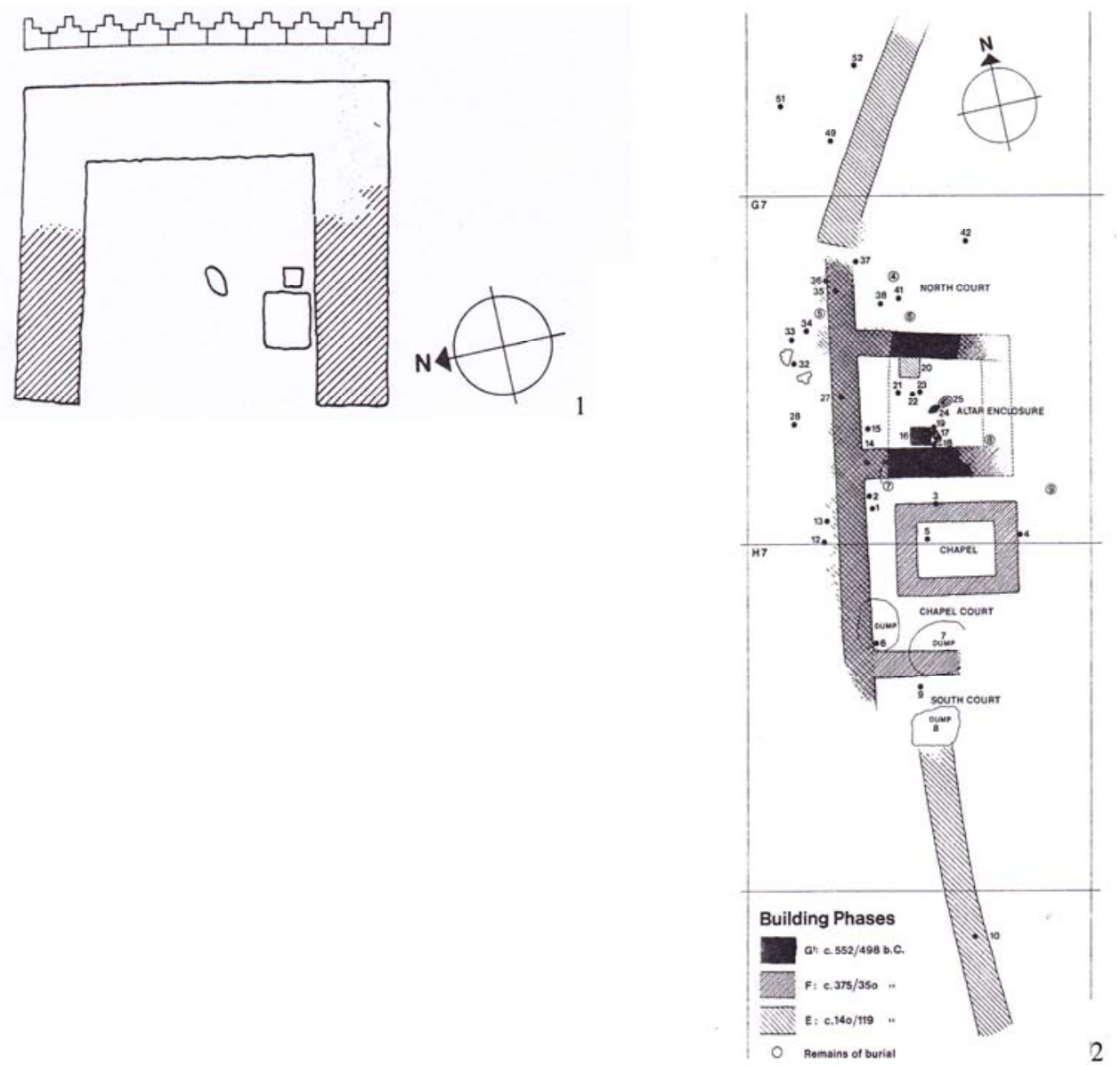

Fig. 9: Tell Sukas, Port Méridional, Periodes G1-F-E; 1, reconstruction de l'Altar Enclosure (Riis 1979, fig. 219); deuxième moitié du VI siècle av. J.-C.; 2, plan schématique du sanctuaire, VI-II siècle av. J.-C. (Riis 1979, fig. 220). 